\title{
35. Jahrestagung der Österreichischen Gesellschaft für Radioonkologie, Radiobiologie und Medizinische Radiophysik
}

\author{
28.-29. September 2018 \\ Salzburg Congress \\ Tagungspräsident: \\ Prim. Univ.-Prof. Dr. Felix Sedlmayer \\ Kongresssekretär: \\ Oberarzt PD Dr. Gerd Fastner \\ Uniklinikum Salzburg, Landeskrankenhaus \\ Universitätsklinik für Radiotherapie und Radio-Onkologie der PMU \\ Veranstalter: \\ Österreichische Gesellschaft für Radioonkologie, Radiobiologie und \\ Medizinische Radiophysik (ÖGRO)

\section{Präsidentin:} \\ Prim. Univ.-Doz. Dr. A.U. Schratter-Sehn

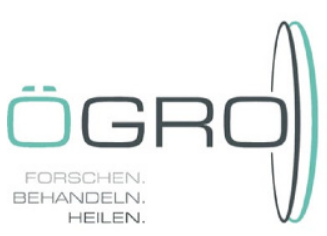 \\ Jahrestagung 2018




\section{Prostata-Karzinom}

\section{PRO-01}

\section{Prostate Cancer Radiotherapy in Austria}

M. Geier ${ }^{1}$, G. Goldner ${ }^{2}$, P. Clemens ${ }^{3}$, M. Gruber ${ }^{4}$, R. Harasleben ${ }^{5}$, T. Langsenlehner ${ }^{6}$, M. Metz ${ }^{7}$, C. Resl ${ }^{8}$, D. H. Seewald ${ }^{9}$, S. Skvortsov ${ }^{10}$, C. Steffal ${ }^{11}$, F. Wolf ${ }^{12}$, E. Nechvile ${ }^{13}$, ÖGRO AK Prostata

${ }^{1}$ Abteilung für Radioonkologie, Ordensklinikum Linz, Linz, Austria ${ }^{2}$ Universitätsklinik für Strahlentherapie, Medizinische Universität Wien, Vienna, Austria

${ }^{3}$ Abteilung für Strahlentherapie und Radio-Onkologie,

Landeskrankenhaus Feldkirch, Feldkirch, Austria

${ }^{4}$ Institut für Strahlentherapie/Radioonkologie, Klinikum Klagenfurt, Klagenfurt, Austria

${ }^{5}$ Institut für Radioonkologie, Sozialmedizinisches Zentrum OstDonauspital Wien, Vienna, Austria

${ }^{6}$ Univesrsitätsklinik für Strahlentherapie-Radioonkologie, LKH-Univ. Klinikum Graz, Graz, Austria

${ }^{7}$ Institut für Radioonkologie-Strahlentherapie, Landesklinikum-

Wiener Neustadt, Vienna, Austria

${ }^{8}$ Abteilung für Strahlentherapie-Radioonkologie,

Universitätsklinikum Krems, Krems, Austria

${ }^{9}$ Institut für Strahlentherapie-Radioonkologie, Salzkammergut

Klinikum Vöcklabruck, Vöcklabruck, Austria

${ }^{10}$ Universitätsklinik für Strahlentherapie-Radioonkologie,

Medizinische Universität Innsbruck, Innsbruck, Austria

${ }^{11}$ Institut für Radioonkologie, Sozialmedizinsches Zentrum Süd-

Kaiser Franz Josef Spital Wien, Vienna, Austria

${ }^{12}$ Uniklinikum Salzburg-Landeskrankenhaus, Univ.-Klinik für

Strahklentherapie und Radio-Onkologie, Salzburg, Austria

${ }^{13}$ Sonderbteilung für Strahlentherapie, Krankenhaus Hietzing, Vienna, Austria

Introduction: Aim of this analysis initiated by the ÖGRO prostate cancer working group was to assess the current status of prostate cancer radiotherapy in Austria and compare these numbers to previous data from 2007 and 2014 to describe the development of radiotherapy treatment to prostate cancer patients.

Methods: A questionnaire was sent to all 14 Austrian departments asking about numbers of prostate cancer patients treated, including the indication of treatment (primary versus postoperative), treatment techniques and volumes (prostate only vs whole pelvis) as well as applied dose ranges and particular treatment concepts. Data investigated were based on the year 2016 .

Results: All 14 centers decided to participate. According to the questionnaires 2401 prostate cancer patients were treated (1698 primary, 703 postoperative) in 2016. Compared to the data of 2007 and 2014, the number of primary patients increased by $46 \%$ and $21 \%$, respectively, whereas the number of patients treated in a postoperative setting only increased by $21 \%$ from 2007 to 2014 and revealed stable or slight decreasing rates from 2014 to 2016.

In 2016 about $90 \%$ of primary treated patients received external beam radiotherapy (EBRT) compared to 10 percent receiving brachytherapy or combination of both. All postoperative patients were treated with EBRT, using volumetric arc radiotherapy in more than $60 \%$ of cases. Regarding primary EBRT doses ranged from 66 to $78 \mathrm{~Gy}$ using normofractionated schedules and 35 to 74 Gy in hypofractionated courses. Hypofractionated radiotherapy at was applied to about $19 \%$ of the patients at 8 departments, at which some departments treated most of the patients using hypofractionated courses and others used hypofractionation only in selected cases. Postoperative radiotherapy in more than $85 \%$ patients was normofractioned and only 3 departments used hypofractionation in this setting. All centers practiced a risk-adapted dose prescription. Additional pelvic lymph node irradiation to primary treated patients was performed at 12 departments and at 13 departments to postoperative patients, respectively. The inclusion of this area was based on risk or evidence of involvement using different stratification procedures or staging techniques.

Conclusion: The national survey could show that an increasing number of prostate cancer patients in Austria is treated with primary or postoperative radiotherapy. If available, IMRT and especially VMAT builds ,standard of care“ for EBRT. Interestingly rates of pelvic lymph node irradiation for patients with risk or evidence of involvement increased since IMRT is available and almost all departments provided this treatment, although there is no clear recommendation by the German S3-guidelines and no clear benefit existing in literature.

PRO-02

\section{Permanente interstitielle Brachytherapie des lokalisierten Prostatakarzinoms: Resultate und Vergleich zur externen Teletherapie - Analyse von 879 Patienten}

\section{G. Goldner}

Universitätsklinik für Strahlentherapie, Medizinische Universität Wien, Wien, Österreich

Einleitung: Patienten mit primär lokalisiertem Prostatakarzinom der Niedrig- und Mittelrisikogruppe, die eine alleinige permanente interstitielle Brachytherapie (iBT) erhielten, wurden hinsichtlich der biochemischen Kontrolle und Neben-wirkungen ausgewertet. Alle Patienten wurden von einem Radioonkologen behandelt. Zusätzlich wurden ein Vergleich zur rein lokalen externen Teletherapie mit 74 Gy bzw. 78 Gy durchgeführt.

Methodik: Zwischen 2004 und 2018 erhielten 385 Patienten eine alleinige iBT bei lokalisiertem Niedrig- (234 Patienten) bzw. MittelRisiko (151 Patienten) Prostatakarzinom (T1-T2,N0×M0x) mittels Jod-125 (145 Gy). Eine begleitende Hormontherapie erhielten 57 Patienten. Die biochemische Kontrollrate (bNED) wurde entsprechend der Phoenix-Defintion (Nadir+2) ermittelt und die Spätnebenwirkungen (gastrointestinal und urogenital) nach EORTC/RTOG Score evaluiert. Die externe Teletherapie (IMRT/VMAT od. 3D-CRT) erfolgte mit 74 Gy bei 333 Patienten bzw. 78 Gy bei 161 Patienten.

Ergebnisse: Das mediane Follow-up betrug 51 Monate (3-157). Ein biochem. Rezidiv trat bei 6/234 Patienten der Niedrigrisikogruppe $(2,5 \%)$ und bei 20/151 Patienten der Mittelrisikogruppe (13\%) auf. In der Niedrigrisikogruppe lagen die resultierende 5- bzw. 10-Jahres bNED-Raten bei $96 \%$ bzw. $92 \%$. In der Mittelrisikogruppe lagen die 5- bzw. 10-Jahres bNED-Raten bei $86 \%$ bzw. $65 \%$. 14/26 Patienten mit biochemischem Rezidiv erhielten eine nochmalige externe Radiotherapie. Grad $\geq 3$ Spätnebenwirkungen fanden sich bei 0/385 Patienten (0\% gastrointestinal) bzw. 17/385 Patienten (4\% urogenital). Der Vergleich zur externen Radiotherapie zeigte sowohl in der Niedrig- als auch in der Mittel-risiko Gruppe keinen signifikanten Unterschied hinsichtlich der biochem. Kontrolle.

Diskussion: Die erzielten Ergebnisse zur iBT des lokalisierten Prostatakarzinoms zeigen einerseits mit der internationalen Literatur vergleichbare, exzellente Resultate bezüglich der bNED-Raten bei geringen Raten an schweren Nebenwirkungen und andererseits vergleichbare Ergebnisse zur externen Radiotherapie. 


\section{PRO-03}

\section{Evaluierung der Patientenzufriedenheit nach 19 Jahren Permanentimplatation der Prostata im Donauspital}

R. Harasleben, K. Poljanc, C. Somay, S. Schuch, W. O. Schmidt, R. Hawliczek

Institut für Radioonkologie, SMZ-Ost Donauspital der Stadt Wien, Wien, Österreich

Einleitung: Seit dem Jahr 1999 wird im Donauspital die Permanentimplantation des lokal begrenzten Prostatakarzinoms mit großem Erfolg durchgeführt. Bis zum Juli 2018 wurden insgesamt 696 Patienten implantiert.

Methodik: Wir entwarfen einen einfachen Fragebogen, welcher 10 Fragen zur generellen Zufriedenheit in Bezug auf die allgemeine Lebensqualität, Beschwerden beim Harnlassen, des Darms und zur Potenz beinhalteten. Außerdem wurde das Vertrauen in den Therapieerfolg mit der Frage nach Wiederentscheidung als auch Weiterempfehlung evaluiert.

Ergebnisse: Zum Zeitpunkt der Auswertung wurden 696 Patienten behandelt. 109 Patienten davon sind verstorben, nach unserem Wissen, sieben Patienten am Prostatakarzinom, das entspricht einer tumorbedingten Sterblichkeitsrate von $1,01 \%$.

Es wurden 600 Fragebögen mit einem Rückkuvert versandt. 52 Kuverts kamen mit dem Vermerk ,verzogen“, ,falsche Adresse“ oder „,verstorben“ zurück. Wir erhielten 404 statistisch auswertbare Fragebögen, was einer Rücklaufquote von 73,72 \% entspricht.

Diskussion: Die hohe Rücklaufrate korreliert unserer Ansicht nach mit der hohen Zufriedenheit der Patienten. Die Ergebnisse dieser Befragung werden präsentiert und bezüglich der standardisierten Fragebögen IPSS, EORTC und IIEF diskutiert.

\section{PRO-04}

Risk adapted moderate hypofractionation of low, intermediate and high risk prostate cancer - feasibility, QOL, acute toxicity and outcome

\section{F. Wolf, A. Schörghofer, F. Sedlmayer}

Universitätsklinik für Radioonkologie und Radiotherapie, Uniklinikum Salzburg-Landeskrankenhaus, Salzburg, Austria

Introduction: Depending on its risk stratification prostate cancer is a highly heterogenous disease with oncologic outcomes ranging from unaltered compared to healthy individuals to becoming a rapidly progressing and ultimately deadly disease.

We therefore aimed to tailor radiation treatment to the risk status by using three different hypofractionated radiation regimen differing in applied dose, use of rectum spacer, inclusion of pelvic lymphnodes and use of androgen deprivation therapy. Here we report on feasibility, acute toxicity, quality of life and outcome at a median follow up of 15 months.

Methods: Acute rectal toxicity was assessed by endosopy and scoring mucosal reactions using Vienna Rectoscopy Score. Acute urinary toxicity was scored using IPSS score. In addition, quality of life was scored using QLQ-PR25 questionnaires and reportet for urinary, bowel and treatment related symptoms as well as sexual functioning.

Results: All treatment regimens were tolerated well. There was no significant difference in any outcome between LR and IR except sexual functioning score, presumably due to the 6 months hormone treatment. In high risk patients, acute GI toxicity was significantly higher, presumably due to the higher rectal dose contributed by the pelvic fields, but reverted to baseline levels at 6 months follow up.

Conclusion: Risk adapted hypofractionated radiation treatment is feasible and tolerated well. Toxicity increases with treatment escalati- on. In our opinion, the slightly higher toxicity in HR patients should be accepted considering their poorer outcome and higher risk of developing metastases which might be prevented by an escalated therapy, in particular by inclusion of pelvic lymphnodes.

\section{PRO-05}

Rectum-spacer related acute toxicity - endoscopy results of $\mathbf{4 0 3}$ prostate cancer patients after implantation of gel or balloon spacers

\section{A. Schörghofer, F. Wolf, F. Sedlmayer}

Universitätsklinik für Radioonkologie und Radiotherapie, Uniklinikum Salzburg-Landeskrankenhaus, Salzburg, Austria

Introduction: Rectal spacers are used to limit dose to the anterior rectal wall in high dose external beam radiation therapy of the prostate and have been shown to reduce radiation induced toxicity. Here we report the complication rate and toxicity of the implantation procedure in a large cohort of patients who have either received a gel- or balloon-type spacer.

Methods: We have analyzed spacer related acute and late rectal toxicity by endoscopy using a mucosa scoring system (Vienna Rectoscopy Score) as well as CTCAE V.4 in a cohort of 403 patients. In addition, in post- implant planning MRIs, position and rotation of balloon spacers were correlated to incidence and grade of rectal reactions.

Results: Overall rectal toxicity was very low with average VRS scores of 0.06 at the day after implantation, 0.10 at the end of RT, 0.31 at 6 months and 0.42 at 12 months follow up. Acute Grade 3 toxicity (rectum perforation and urethral damage) directly related to the implantation procedure occurred in $1.49 \%(n=6)$ and was seen exclusively in patients who had received the spacer balloon. Analysis of post implant MR imaging did not identify abnormal or mal-rotated positions of this spacer to be a predictive factors for the occurrence of spacer related G3 toxicities.

Conclusion: Spacer technology is an effective means to minimize dose to the anterior rectal wall. However, the benefits in terms of dose sparing need to be weighed against unlikely, but possible risks of complications such as rectum perforation.

\section{PRO-06}

\section{Postoperative Radiotherapie des Prostatakarzinoms - Konventionelle vs. Hypo-Fraktionierung: Analyse von Nebenwirkungen bei 691 Patienten}

\section{G.Goldner}

Universitätsklinik für Strahlentherapie, Medizinische Universität Wien, Wien, Österreich

Einleitung: Insgesamt sind 691 postoperative Patienten mit Prostatakarzinom (pT2-3; pN0x; M0x), die eine adjuvante bzw. salvage Radiotherapie erhielten, in der Auswertung eingeschlossen.

Methodik: Die Radiotherapie erfolgte bei 521 Patienten konventionell mit einer Dosis von 66,6 Gy (60-74 Gy) bei 1,8 bzw. 2 Gy Einzeldosis als 4 Felder Becken Box Technik. Davon wurden 348 Patienten im Bereich der Prostataloge bestrahlt und 173 Patienten im Bereich der Prostataloge und Beckenlymphabflusswege behandelt.

Bei 170 Patienten erfolgte die Bestrahlung hypofraktioniert mit einer Dosis von 62,5 Gy (60-63,75 Gy) bei 2,5 bzw 2,55 Gy Einzeldosis mittels VMAT Technik. Davon wurden 69 Patienten im Bereich der Prostataloge bestrahlt und 101 Patienten im Bereich der Prostataloge und Beckenlymphabflusswege behandelt. 
Akut und Spätnebenwirkungen nach EORTC/RTOG der konventionell-fraktionierten Patienten (konRad-Gruppe) vs. der Hypofraktionierten Patienten (hypRad-Gruppe) wurden verglichen.

Ergebnisse: Das mittlere Follow-up betrug 68 Monate (3-252 Mo) in der konRad-Gruppe und 20 Monate (3-49 Mo) in der hypRad-Gruppe.

Bei Patienten mit alleiniger Prostatalogen Bestrahlung fanden sich maximale akute Nebenwirkungen in der konRad-Gruppe bzw in der hypRad-Gruppe mit:

Rektal: Grad 0: $28 \%$ bzw. $36 \%$; Grad 1: $47 \%$ bzw. $48 \%$; Grad $\geq 2: 25 \%$ bzw. $16 \%$

Urogenital: Grad 0: $40 \%$ bzw. $41 \%$; Grad 1: $45 \%$ bzw. $49 \%$; Grad $\geq 2: 16 \%$ bzw. $10 \%$

Und späte Nebenwirkungen in der konRad-Gruppe bzw in der hypRad-Gruppe mit:

Rektal: Grad 0: $64 \%$ bzw. $73 \%$; Grad 1: $22 \%$ bzw. $16 \%$; Grad $\geq 2: 14 \%$ bzw. $12 \%$

Urogenital: Grad 0: $52 \%$ bzw. $59 \%$; Grad 1: $28 \%$ bzw. $20 \%$; Grad $\geq 2: 20 \%$ bzw. $20 \%$

Bei Patienten mit Prostatalogen und Beckenlymphabfluss Bestrahlung fanden sich maximale akute Nebenwirkungen in der konRadGruppe bzw in der hypRad-Gruppe:

Rektal: Grad 0: $13 \%$ bzw. $20 \%$; Grad 1: $43 \%$ bzw. $50 \%$; Grad 22: $45 \%$ bzw. $31 \%$

Urogenital: Grad 0: $27 \%$ bzw. $43 \%$; Grad 1: $54 \%$ bzw. $46 \%$; Grad $\geq 2: 20 \%$ bzw. $16 \%$

Und späte Nebenwirkungen in der konRad-Gruppe bzw in der hypRad-Gruppe mit:

Rektal: Grad 0: $57 \%$ bzw. $74 \%$; Grad 1: $32 \%$ bzw. $22 \%$; Grad $\geq 2: 11 \%$ bzw. $4 \%$

Urogenital: Grad 0: $60 \%$ bzw. $64 \%$; Grad 1: $20 \%$ bzw. $26 \%$; Grad $\geq 2: 20 \%$ bzw. $10 \%$

Diskussion: Die Hypofraktionierung mittels VMAT Technik zeigte weniger akut Nebenwirkungen - rektal und urogenital - sowohl bei alleiniger Prostatalogenbestrahlung als auch bei Inklusion der Beckenlymphabflusswege.

Hinsichtlich der Spät-Nebenwirkungen zeigte sich vor allem bei Inklusion der Beckenlymphabflusswege ein Vorteil für die Hypofraktionierung mit VMAT Technik.

\section{Mamma-Karzinom}

\section{MAM-01}

\section{Bestrahlung in tiefer Inspiration bei linksseitigem Brustkrebs - Ein Zwischenbericht der SAVE HEART Studie}

S. Corradini ${ }^{1}$, S. Schönecker ${ }^{1}$, A. Gaasch ${ }^{1}$, M. Pazos ${ }^{1}$, D. Reitz ${ }^{1}$, M. Braun ${ }^{2}$, N. Harbeck ${ }^{3}$, U Ganswindt $^{1,4}$, M. Niyazi ${ }^{2}$, C. Belka ${ }^{1}$

${ }^{1}$ Klinik für Strahlentherapie, LMU München, München, Deutschland ${ }^{2}$ Brustzentrum Rotkreuzklinikum, München, Deutschland

${ }^{3}$ Brustzentrum LMU München, München, Deutschland

${ }^{4}$ Klinik für Strahlentherapie, Medizinsche Universität Innsbruck, Innsbruck, Österreich

Einleitung: SAVE-HEART ist eine prospektive Studie zur linksseitigen Brustbestrahlung in tiefer Inspiration (DIBH). Neben der Erfassung der kardialen Baseline-Risiken der Brustkrebspatientinnen, wird der dosimetrische Benefit durch die DIBH dokumentiert. Die Studie ist im Deutschen Register für klinische Studien unter der Studiennummer DRKS00011213 registriert. Wir berichten nachfolgend über die dosimetrischen Auswertungen.

Methodik: Einschlusskriterien waren ein histologisch gesichertes linksseitiges invasives Mammakarzinom oder Carcinoma in situ nach brusterhaltender Operation oder Mastektomie mit Indikation zur ad- juvanten Radiotherapie der Restbrust/Thoraxwand \pm lokoregionäre Lymphabflusswege, sowie die Fähigkeit den Atem für mindestens 20 Sekunden anhalten zu können. Eine Hypofraktionierung (40,05 Gy in 15 Fraktionen) oder Boostbestrahlung waren erlaubt. Insgesamt wurden bisher 208 Patientinnen gescreent und 175 Patientinnen innerhalb der Studie in DIBH bestrahlt. Die Bestrahlung in tiefer Inspiration wurde mittels automatisch getriggertem Oberflächensystem CatalystTM/SentinelTM (C-RAD AB, Uppsala, Schweden) mit Audio- und WLAN-Brillen-basiertem Videofeedback durchgeführt. CT und Oberflächendaten wurden sowohl in DIBH als auch in freier Atmung (FB) akquiriert.

Ergebnisse: Die mediane Nachbeobachtungszeit beträgt derzeit ca. 3 Monate. 77 von 175 Patienten sind normofraktioniert bestrahlt worden. Für diese Patienten konnte die mittlere Herzdosis durch DIBH um $-40,02 \%$ (2,91 auf 1,74 Gy) reduziert werden. Die Maximaldosis des Herzens und der LAD (left anterior descending artery) konnte um $-36,21 \%$ (45,45 auf 28,99 Gy) bzw. $-52,61 \%$ (35,10 auf 16,63 Gy) gesenkt werden. Für die 98 hypofraktioniert bestrahlten Patienten, ergaben sich folgende Reduktionen durch tiefe Inspiration: Mittlere Herzdosis $-39,66 \%(2,12$ Gy [EQD2 $(\alpha / \beta=3 \mathrm{~Gy}): 2,40 \mathrm{~Gy}$ ] auf 1,28 Gy [EQD2: 1,45 Gy]); Maximaldosis Herz -38,98 \% (35,08 Gy [EQD2: 39,78 Gy] auf 21,41 Gy [EQD2: 24,28 Gy]), Maximaldosis LAD -52,53 \% (27,12 Gy [EQD2: 30,75 Gy] auf 12,87 Gy [EQD2: $14,59 \mathrm{~Gy}]$ ). Auch für die Lunge zeigte sich in DIBH eine Verbesserung mit einer Reduktion der mittleren Lungendosis $(-12,29 \%$, von 7,63 Gy auf $6,69 \mathrm{~Gy})$ als auch der V20 (-18,34 \%, von $14,51 \mathrm{~Gy}$ auf 11,84 Gy). Alle hier beschriebenen Werte waren für ein Signifikanzniveau $p<0,05$ signifikant.

Diskussion: Insgesamt zeigen unsere dosimetrischen Daten eine signifikante Herzschonung bei linksseitiger Brustbestrahlung. Nach unserer Einschätzung sollten alle linksseitigen Brustkrebspatientinnen mit Indikation zur Bestrahlung auf eine potentielle Therapie in tiefer Inspiration gescreent und entsprechend therapiert werden.

\section{MAM-02}

\section{Kremser Atemanhaltetechnik zur Schonung des Herzens bei Patientinnen mit linksseitigem Mammakarzinom}

K. Lövey, M. Schiebl, C. Naschenweng, N. Metz, J. Ditz, P. Ladinig, K. Raditsch, M. Seel, A. Bayerl

Abteilung für Strahlentherapie-Radioonkologie, UK Krems, Krems, Österreich

Einleitung: Verbesserung der Dosisbelastung der linken anterior descendenten Koronararterie (LAD) durch Anwendung einer einfachen Atemanhaltetechnik (AA) sowie Evaluierung von Kriterien für die Vorhersage eines Benefits durch die AA.

Methodik: Die Daten der im UK Krems zwischen 05/16-08/17 mit linksseitigem Mammakarzinom bestrahlten 100 Patienten wurden ausgewertet. Bei jenen, die den Atem gut anhalten konnten, wurde ein CT in AA und ein CT in freier Atmung (FA) durchgeführt, an beiden CTSätzen konturiert, Pläne angefertigt und die Pläne in AA und FA miteinander verglichen. Die dosimetrischen Daten, der Einfluss des Tumorsitzes und der Patienten bezogenen Faktoren wie Alter, Body Mass Index (BMI), Begleiterkrankungen, sowie der maximalen Herzdistanz (MHD) und zentralen Lungendistanz (CLD) auf die Wirksamkeit der AA wurden ausgewertet.

Ergebnisse: Bei 62 Patienten wurde ein Planungs-CT in AA durchgeführt, 30 Patienten konnten aufgrund Kurzatmigkeit den Atem nicht anhalten, weitere 8 hatten Verständnisprobleme oder die AA abgelehnt. Bei $84 \%$ der 62 Patienten, die den Atem gut anhalten konnten, war der AA-Plan als klinisch signifikant besser gefunden, bei 10 Patienten hat diese Technik keinen Vorteil gebracht. Die AA war bei $81 \%$ der Patienten mit cranialem Tumorsitz vorteilhaft. Die kurzatmigen Patienten 
waren älter (67 vs. 58 Jahre), adipös (BMI 30,1 vs. 26,3), hatten häufiger relevante Herzkrankheiten (17\% vs. $3 \%$ ). Der Unterschied der MHD in AA und FA für die letztlich in AA behandelten 52 Patienten war signifikant größer, als bei den 10 Patienten, die von der AA nicht profitierten (9,8 vs. $2,5 \mathrm{~mm}$ ). Der Unterschied der MHD in FA für die 2 Patientengruppen war nicht relevant (13,8 vs. 11,5 mm). Die CLD war ebenfalls nicht als Vorhersage-Faktor verwendbar.

Diskussion: Die AA bringt bei 5/6 der Patienten mit linksseitigem Mammakarzinom einen signifikanten Vorteil. Dieser Vorteil ist unabhängig vom Tumorsitz nachweisbar. Durch die Messung der MHD/ CLD Werte allein in FA konnte nicht vorhergesagt werden, welcher Patient von einer AA profitieren würde. Daher wird aktuell der Unterschied der MHD-Werte in FA und AA als mögliches Kriterium für die Vorhersage eines Benefits geprüft und in der Folge ausgewertet werden. Bei jenem Drittel der Patientinnen, die den Atem nicht ausreichend anhalten konnten, aber prinzipiell von einer AA profitieren könnten, wird die Entscheidung über die Verwendung einer alternativen herzschonenden Technik individuell getroffen.

\section{MAM-03}

\section{Advanced locally recurrent breast cancer (LRBC): the potential of re-irradiation and thermography-controlled superficial wIRA-hyperthermia}

\section{Notter ${ }^{1}$, P. Vaupel ${ }^{2}$}

${ }^{1}$ Abteilung für Radio-Onkologie Lindenhofspital, Bern, Switzerland ${ }^{2}$ Klinik und Poliklinik für Radioonkologie und Strahlentherapie, Klinikum rechts der Isar, Technische Universität München, München, Germany

Introduction: Superficial hyperthermia (sHT) combined with RT has proven efficacy in the management of LBRC. However, the treatment of widespread recurrences is limited using currently available microwave sHT. The implementation of thermography-controlled water-filtered infrared A hyperthermia (wIRA-HT) offers the possibility to treat very extended LRBC achieving local control even with lower RT doses (Notter et al., IJH, 2017).

Methods: This updated retrospective analysis includes a total of 140 patients with pre-irradiated LRBC, subsequently treated with combined hypofractionated re-RT of 20 Gy total dose $(5 \times 4$ Gy, 1x/week), delivered 1-4 min after wIRA-HT. 27 patients presented microscopic disease, 113 with macroscopic disease. Staging of macroscopic disease was defined as follows: Stage $\mathrm{I}=$ tumor $<10 \mathrm{~cm}$, stage $\mathrm{II}=$ restricted to homolateral chest wall; stage III = beyond homolateral chest wall, stage IV = III + extension to the back (cancer en cuirasse). 84/113 (74\%) showed large extension (stages II - IV).

Results: Response rates of macroscopic diseases: Stage I=79\% CR, $21 \%$ PR. Stage II $=53 \%$ CR, 44\% PR, 3\% NC. Stage III $=37 \%$ CR, $59 \%$ PR, $4 \%$ PD. Stage IV $=86 \%$ PR, $14 \%$ NC. Local control (LC) during lifetime after CR was obtained in $63 \%$, local stabilisation after PR in $43 \%$. Microscopic disease remained controlled in $96 \%$ of the patients. Due to low toxicity re-recurrences could be re-treated using the same protocol. No thermal skin damage $\geq$ grade 2 was observed. Conclusion: Response rates are greatly dependent on LRBC extension. Our data show a substantial change in LC at stage IV with no patient achieving CR. However, palliation with local stabilisation was seen in $56 \%$ of these patients. The contact-free heating technique (hydrosun ${ }^{\circledR}-\mathrm{TWH} 1500$ ) offers a series of advantages especially for the treatment of irregularly shaped, widespread, ulcerated lesions. Realtime thermography can assess large surface temperature distributions and by automatic control can avoid hot spots and grade $2-4$ skin toxicities (Vaupel et al., IJH, 2018).
Cancer en cuirasse remains a very challenging disease. However, even these palliative patients can benefit from sHT immediately followed by low dose re-RT.

\section{MAM-04}

\section{Molekulare Resistenzmechanismen von invasiven Mammakarzinomzellen gegen ionisierende Strahlung}

B. Aschenbrenner ${ }^{1}$, G. Negro' ${ }^{1}$ S. Skvortsov ${ }^{1}$, S. Kranjc ${ }^{2}$, M. Čemažar ${ }^{2}$, U. M. Ganswindt ${ }^{1}$, I. I. Skvortsova ${ }^{1}$

${ }^{1}$ EXTRO-Lab, Dept. of Therapeutic Radiology and Oncology, Medical University Innsbruck, Innsbruck, Österreich ${ }^{2}$ Institute of Oncology, University of Ljubljana, Ljubljana, Slowenien

Einleitung: Mammakarzinom-Metastasen sind schwer behandelbar und meist verantwortlich für den Tod der Patientinnen mit diagnostiziertem Mammakarzinom (Mk). Radiotherapie (RT) ist eine weit verbreitete Methode um Metastasen zu behandeln, jedoch ist die Effizienz von konventioneller RT oft nicht befriedigend, da Resistenzen gegenüber ionisierender Strahlung auftreten. Es gibt den Verdacht, dass invasive Zellen über eine intrinsische Resistenz gegenüber Strahlung besitzen. Um dies zu untersuchen wurden Mammakarzinomzellen mit erhöhter Invasivität aus Mammakarzinom Zelllinien (Mkz) isoliert und mit ihren parentalen Mammakarzinom Zelllinien (pMkz) verglichen.

Methodik: Invasive Mammakarzinomzelllinien (iMkz) wurde durch wiederholte Wanderung von pMkz (MDA-MB-231(dreifach negativ), T47D (hormonabhängig) und Au565 (Her2 positiv)) durch eine Boyden-Kammer erhalten. Die Invasivität wurde mittels Invasions-Assay (Kollagen I und Laminin) getestet. Um Proteomik Daten zu erhalten wurden Zelllysate der Mkz an MyOmix Dx gesendet und für uns generiert. In vivo Tumorformierung der Mkz in Mäusen wurde von unserem Kollaborationspartner in Ljubljana untersucht. Die Reaktion der Mkz auf ionisierende Strahlung wurde mittels Western blot, Einzelzellgelelektrophorese, Durchflusszytometrie und durch Mikroskopie an lebenden Zellen untersucht.

Ergebnisse: Es wurden erfolgreich drei Mkz mit erhöhter Invasivität etabliert: diese haben ein erhöhtes Potential durch Kollagen I und Laminin zu wandern.

Diese iMkz haben eine große Zahl an hochregulierten Proteinen welche im Zusammenhang mit Strahlentherapieresistenz und Invasion stehen.

Dreifach negative pMkz (MDA-MB-231) sind von sich aus sehr resistent gegen Strahlung und besitzen eine hohe Invasivität: das Selektionieren und somit die Erhöhung der Invasivität hat nur gering die Radioresistenz erhöht.

Hormon abhängige pMkz (T47D) zeigen eine geringe Invasivität auf und reagieren wesentlich stärker auf Strahlung: diese induzieren vermehrt Apoptose und haben größere DNA Schäden nach der Bestrahlung im Vergleich zu dreifach negativen Mkz. Die invasiven T47D Zellen sind wesentlich resistenter als deren pMkz gegen ionisierende Strahlung.

Die Her2 positiven Mkz (Au565) zeigen ein gegenteiliges Verhalten: Die iMkz haben zwar das höchste Invasionspotential von allen 6 Mkz sind aber nicht resistenter gegen ionisierende Strahlung als ihren pMkz.

Diskussion: Es gibt einen Zusammenhang zwischen Invasionspotential und Strahlentherapieresistenz, aber generalisieren lässt es sich nicht. Die Ergebnisse deuten jedenfalls auf eine heterogene Bewältigungsstrategie von Mkz mit ionisierender Strahlung hin: z. B.: Caspase-3 induzierter Zelltod spielt nur in parentalen MDA-MB-231 und Au565 eine Rolle. Autophagie Induzierung nach der Bestrahlung ist in allen $6 \mathrm{Mkz}$ verschieden reguliert, nur die parentalen T47D Zellen weisen eine hohe Apoptose rate auf nach der Bestrahlung mit 2 Gray ionisierender Strahlung. 


\section{Varia}

\section{VAR-01}

\section{RADIOMICS - airy-fairy gimmick for physicists or valuable tool for clinicians?}

M. Daniel ${ }^{1,2}$, P. Kuess ${ }^{1,2}$, P. Andrzejewski ${ }^{1,3}$, T. Nyholm ${ }^{4}$, T. Helbich ${ }^{1}$, F. Dragschitz ${ }^{2}$, G. Goldner ${ }^{2}$, S. Polanec ${ }^{1,5}$, P Baltzer $^{1,5}$, D. Georg ${ }^{1,2}$

${ }^{1}$ Department of Radiotherapy, Comprehensive Cancer Center, Medical University of Vienna/AKH Vienna, Vienna, Austria ${ }^{2}$ Department of Radiotherapy, Medical University of Vienna, Vienna ${ }^{3}$ Department of Medical Physiscs, Medical University of Vienna/ AKH Vienna, Vienna, Austria

${ }^{4}$ Department for Radiation Sciences, Radiation Physics, Umeå University, Umeå, Sweden

${ }^{5}$ Department of Biomedical Imaging and Image-guided Therapy, Medical University of Vienna, Vienna, Austria

Introduction: The current hype around radiomics in the area of clinical radiology is reflected by the exponential increase of publications based on this approach. The fascination on this topic is driven by the believe that hidden quantifiable information is integral part of the "digital" images, which could potentially be utilized for diagnostic and/ or therapeutic purposes. This contribution is a first-hand experience report of several years of research within the Christian Doppler Laboratory for Medical Radiation Research for Radiation Oncology at the Medical University of Vienna to address the following questions: Are the developments in the field of radiomics transferable to current clinical praxis? What can the combination of multiparametric imaging and radiomics offer to advanced radiation oncology practice?

One of our most recent studies shall serve as an example to discuss the impact and relevance of radiomics.

Methods: The retrospective, IRB-approved study was motivated by the rising interest in dose painting approaches that aim to boost the intraprostatic lesion in patients with prostate cancer. With an additional dose to this region, an increasing probability of tumour control is anticipated. The value of a textural feature essay extracted from multiparametric MRI to define boost volumes was investigated. 28 prostate cancer patients who underwent T2w- and DWI-MRI were analyzed. Lesions, central gland and peripheral zone were delineated by an experienced urogenital radiologist based on imaging findings and localized transrectal ultrasound guided biopsy results. Histogram parameters and 20 Haralick textural features (TF) were calculated and tested for their ability to distinguish benign from malignant prostatic tissue.

Results: TF outperformed conventional histogram parameters in distinguishing healthy from tumour tissue both regarding sensitivity and specificity. The TF approach has the potential to be used for quantitative image-assisted boost volume delineation.

Conclusion: From our experience, the radiomics approach has definitely the potential to be implemented as versatile tool into clinical practice. Its applicability for diagnostic and prognostic purposes includes autosegmentation of lesions and treatment response assessment. However, accessible software tools still need to be developed. The interdisciplinary nature of this field calls for close collaboration of physicists, clinicians, radiologists and software engineers.

\section{VAR-02}

\section{Retrospektive Single-Center Auswertung der Radiochirurgie von Hirnmetastasen}

J. Mangesius ${ }^{1}$, C. Arnold ${ }^{1}$, C. Freyschlag ${ }^{2}$, D. Vasiljevic ${ }^{1}$, I. Kvitsaridze ${ }^{1}$, P. Lukas ${ }^{1}$, U. Ganswindt ${ }^{1}$, M. Nevinny-Stickel ${ }^{1}$
${ }^{1}$ Universitätsklinik für Strahlentherapie-Radioonkologie, Medizinsche Universität Innsbruck, Innsbruck, Österreich

${ }^{2}$ Universitätsklinik für Neurochirurgie, Medizinsche Universität Innsbruck, Innsbruck, Österreich

Einleitung: Hirnmetastasen sind die häufigste Form maligner Tumoren im Gehirn bei Erwachsenen. Etwa 10-40 \% aller Tumorpatienten entwickeln im Verlauf Hirnmetastasen. Sie sind mit einer deutlich schlechteren Prognose verbunden und können eine schwerwiegende Beeinträchtigung der Lebensqualität zur Folge haben. Lungenkrebs, Brustkrebs und Melanom zählen zu den häufigsten Ursachen und sind zusammen für etwa $70 \%$ aller Hirnmetastasen verantwortlich. $\mathrm{Zu}$ den möglichen Therapieoptionen zählen Resektion, Ganzhirnbestrahlung sowie die fraktionierte oder einzeitige Radiochirurgie.

Methodik: Wir berichten die Ergebnisse einer retrospektiven SingleCenter Studie von 108 Patienten mit 213 Metastasen, welche zwischen 08/2003 und 03/2018 an der Universitätsklinik für StrahlentherapieRadioonkologie Innsbruck bei Hirnmetastasen eine einzeitige LINACbasierte Radiochirurgie erhielten. Wir analysierten die Daten hinsichtlich des Gesamtüberlebens sowie der lokalen Progressionsrate und des lokalen progressionsfreien Überlebens anhand der Kaplan-Maier Methode. Es wurde die Validität unterschiedlicher Risikoscores in unserem Patientenkollektiv überprüft. Zudem wurde der Einfluss des PTV auf das Risiko einer lokalen Progression untersucht.

Ergebnisse: Das mediane follow-up betrug 11 Monate (range 0-99). Die mediane Überlebenszeit nach der ersten SRS betrug 13 Monate (range 0-100) und das Gesamtüberleben nach 1 und 2 Jahren 50,5\% und 32,5\%. Die lokale Kontrollrate betrug $89,7 \%$. Das lokale progressionsfreie Überleben nach einem Jahr betrug 90,9\% und nach zwei Jahren $89,9 \%$. Ein PTV von $>1 \mathrm{~cm}^{3}$ war mit einer statistisch signifikanten Verschlechterung der lokalen Kontrollrate $(p=0,004)$ sowie des PFS $(p=0,001)$ verbunden.

Es wurde die Validität verschiedener Risikoscores zur Prädiktion des OS in unserem Patientenkollektiv überprüft. Die höchste Korrelation wurde mit BSBM (basic score for brain metastases) und RPA Klassifizierung erzielt (Spearman Korrelation, $p<0,001)$, eine schwache Korrelation bestand bei SIR (score index for radiosurgery) $(p=0,001)$.

Es wurde ein signifikant besseres OS bei Frauen als bei Männern festgestellt, dieser Unterschied blieb auch nach Exklusion der Mammakarzinome bestehend $(p=0,01)$

Diskussion: Die einzeitige Radiochirurgie bietet eine sichere und effektive Therapieoption für die Behandlung von singulären oder multiplen Hirnmetastasen und erzielt eine sehr hohe lokale Kontrollrate.

\section{VAR-03}

\section{Unconventional SBRT-based partial tumor irradiation targeting hypoxic segment of unresectable bulky tumors exploiting the bystander and abscopal effects}

\section{S. Tubin}

Institut für Strahlentherapie/Radioonkologie, Klinikum Klagenfurt, Klagenfurt, Austria

Introduction: Previously, our pre-clinical studies on focused bystander (BE)/abscopal effect (AE)-induction, have shown for the first time that high-dose partial (vs. whole) tumor irradiation, targeting exclusively hypoxic (vs. normoxic) tumor resulted in significant radiation-hypoxia-induced bystander and abscopal effects. Those findings were translated to clinic leading to development of our novel SBRT approach for partial tumor irradiation (PTI). We hypothesized that highdose irradiation of exclusively hypoxic tumor segment would generate effective anti-tumor abscopal-signaling and antigen release leading to immune-mediated regression of whole partially-irradiated bulky (due to $\mathrm{BE}$ ) but also of unirradiated distant (metastatic) lesions (due to $\mathrm{AE}$ ). Primary endpoints were $\mathrm{BE}$ and $\mathrm{AE}$ response rates. Secondary end- 
points included overall survival (OS), progression-free survival (PFS), symptom-control rate, assessment of toxicity.

Methods: This study involved 32 oligometastatic patients whose bulky tumors of the lung, head and neck, pancreas, kidney, skin, adrenal glands and lymph nodes were partially irradiated. We defined „Bystander Tumor Volume (BTV)“ (hypoxic segment) using PET and contrast-enhanced CT, as a hypovascularized-hypometabolic junctional zone between the central necrotic and peripheral hypermetabolic tumor segment. Based on tumor site and volume, BTV was irradiated with $1-3$ fractions of 10 or 12 Gy prescribed to $70 \%$ isodose. No patient received chemotherapy or immunotherapy.

Results: On an average, targeted BTV corresponded to $30 \%$ of whole bulky. With median follow-up of 11 months (range: 2-22) OS and PFS were $70 \%$ and $87 \%$, respectively. In $20 \%$ of patients disease progression occurred at distance after mean time to tumor progression of 5 months. Only 1 patient had progression at bulky site (BE rate 97\%). Average bulky shrinkage was $60 \%$ (range: $40-100 \%$ ) with 4 complete responses. AE rate was $51 \%$ with mean reduction of unirradiated metastases of 50\% (range: $30-100 \%$ ). No patient experienced acute or late toxicity of any grade.

Conclusion: SBRT-PTI for bulky tumors was feasible, effective and safe, showing high potential for induction of $\mathrm{BE}$ and $\mathrm{AE}$, resulting in improved local control and prolonged PFS. Relevance: conversion of unresectable lesions into resectable (palliative treatment into curative) as a neoadjuvant option, more suitable (1-3 days) treatment for symptomatic patients in very poor general condition, better cost/effectiveness-profile, no toxicity (very safe re-irradiation).

\section{VAR-04}

\section{Radiochemotherapie und bild-gestützte adaptive Brachytherapie für primäre Vaginalkarzinome: Therapieergebnisse einer internationalen multizentrischen Studie}

M. Schmid ${ }^{1}$, H. Westerveld ${ }^{2}$, R. Nout ${ }^{3}$, C. Chargari ${ }^{4}$, N. Nesvacil ${ }^{1}$, R. Mazeron ${ }^{4}$, L. Fokdal ${ }^{5}$

${ }^{1}$ Universitätsklinik für Strahlentherapie, Medizinische Universität Wien, Wien, Österreich

${ }^{2}$ Department of Radiotherapy, Academic Medical Centre, University of Amesterdam, Amsterdam, Niederlande

${ }^{3}$ Department of Clinical Oncology, Leiden University Medical Centre, Leiden, Niederlande

${ }^{4}$ Radiotherapy Department, Brachytherapy Unit, Gustave Roussy

Cancer Campus, Villejuif, Frankreich

${ }^{5}$ Department of Oncology, Aarhus University Hospital, Aarhus, Dänemark

Einleitung: Retrospektive Analyse der Therapieergebnisse nach Radiochemotherapie und CT-oder MRT gestützter adaptiver Brachytherapie (IGABT) bei primären Vaginalkarzinomen

Methodik: 148 konsekutive Patientinnen aus 5 europäischen Zentren, die im Zeitraum von 2001-2016 mit IGABT behandelt wurden, konnten in die Studie eingeschlossen werden. Patienten-, Tumor- und Behandlungscharakteristika wurden retrospektiv erhoben. Nebenwirkungen wurden nach CTCAE v3.0 graduiert. Die statistische Auswertung erfolgte mit Hilfe der Kaplan-Meier Methode und des Log-rank Test. Ergebnisse: Das mediane Alter betrug 63 Jahre (24-89). Folgende Tumorstadien wurden festgestellt: T1: $n=28(19 \%)$, T2: $n=79(53 \%)$, T3: $n=21(14 \%)$, T4: 20 (14\%). Bei 46 Patientinnen (31\%) wurden regionäre Lymphknoten-Metastasen diagnostiziert.

$98 \%$ der Patientinnen erhielten eine Teletherapie im Bereich des Beckens (45-50.4 Gy). $64 \%$ erhielten eine konkomitante wöchentliche Chemotherapie mit Cisplatin.
Die Brachytherapie erfolgte bei $78 \%$ im PDR-Verfahren und bei $55 \%$ als kombiniert intrakavitäre/interstitielle Applikation. Die Brachytherapie-Dosisplanung erfolgte bei $52 \%$ mit MRT, $28 \%$ mit CT und $19 \%$ mit MRT+CT. Das mediane CTV zum Zeitpunkt der Brachytherapie betrug $17.6 \mathrm{~cm} 3(0-71)$ und die mediane kumulative D90 für das CTV (Teletherapie + Brachytherapie) betrug 80 Gy (52-110; in EQD2).

Nach einem medianen Follow-up von 29 Monaten (3-167) war die lokale Tumorkontrolle $82 \%$, das krankheitsspezifische Überleben $75 \%$ und das Gesamtüberleben $68 \%$. Grad 3 und 4 Nebenwirkungen im Bereich der Harnblase/Urethra, Darm und Vagina wurden bei $8.1 \%, 3 \%, 8.1 \%$ festgestellt.

Eine Dosis-Wirkungsbeziehung im Sinne einer signifikant verbesserten lokalen Tumorkontrolle bei Applikation einer D90>80 Gy konnte für T2-4 Tumoren nachgewiesen werden.

Diskussion: Die IGABT führt bei primären Vaginalkarzinomen zu einer verbesserten lokalen Tumorkontrolle im Vergleich zu den Studien mit Röntgen-gestützter Brachytherapie. Eine internationale prospektive Beobachtungsstudie zu diesem Thema wird voraussichtlich 2019 starten.

\section{VAR-05}

\section{Therapieergebnisse nach Radiochemotherapie und bildgestützter Brachytherapie für Analkanalkarzinome}

M. Heilmann ${ }^{1}$, R. Schmid ${ }^{1}$, C. Kirisits ${ }^{1}$, D. Berger ${ }^{1}$, N. Nesvacil ${ }^{1}$, H. Herrmann ${ }^{1}$, R. Pötter ${ }^{1,2}$, J. Widder ${ }^{1}$, M. Schmid

${ }^{1}$ Universitätsklinik für Strahlentherapie, Medizinische Universität Wien, Wien, Österreich

${ }^{2}$ Christian Doppler Labor für Medizinische Strahlenforschung für die Radioonkologie, Wien, Österreich

Einleitung: Retrospektive Analyse einer Kohorte bestehend aus PatientInnen mit Analkanalkarzinom, die mit Teletherapie mit/ohne Chemotherapie und einer bildgestützten PDR-Brachytherapie (PDR-BT) behandelt wurde.

Methodik: Inkludiert wurden alle PatientInnen mit einem Plattenepithelkarzinom des Analkanals, die für eine primäre Behandlung oder nach inkompletter Resektion von 2000-2017 an unsere Abteilung zugewiesen wurden und einen PDR-BT Boost erhielten. Das Therapiekonzept sah eine Teletherapie auf das kleine Becken bis 46 Gy und einer konkomitanten Chemotherapie bestehend aus Mitomycin-C und Capecitabine oder 5-FU vor. Anschließend erfolgte ein PDR-BT Boost. Einschlusskriterien für eine PDR-BT waren Tumoren, die vor Therapiebeginn weniger als die Hälfte der Zirkumferenz des Analkanals und weniger als $5 \mathrm{~cm}$ Länge einnahmen. Dokumentierte Toxizität wurde entsprechend der CTCAE v4 retrospektiv klassifiziert.

Ergebnisse: Es wurden 52 PatientInnen $(n=46$ Frauen) mit PDRBT behandelt. Das mediane Alter war 62 Jahre. Die Tumoren wurden wie folgt klassifiziert: T1: $n=11(21 \%), \mathrm{T} 2: n=31(60 \%)$, T3: $n=9$ (17\%), T4: $n=1(2 \%)$. Bei 15 PatientInnen fanden sich initial klinisch positive Lymphknoten.

Die mediane Dosis der Teletherapie war 46 Gy (26-50 Gy). Die geplante minimale Zieldosis der PDR-BT war 14,5 Gy ( \pm 2,0 Gy). Eine konkomitante CHT erhielten 42 PatientInnen (81\%). Die mediane Gesamtbehandlungszeit war 53 Tage (42-115 Tage). Die bildgestützte Planung für die Brachytherapie erfolgte bei 15 Patienten mittels MRT (29\%) und bei 37 PatientInnen mittels CT (71\%).

Nach einer medianen Nachbeobachtungszeit von 37 Monaten wurden zwei persistierende Tumoren, ein Lokalrezidiv und eine Fernmetastasierung beobachtet. Das Gesamtüberleben nach 3 Jahren war $85 \%$, die lokale Kontrolle $93 \%$ und das Stoma-freie Überleben $91 \%$. Therapieassoziierte Grad 3-Toxizität trat bei 11 PatientInnen (19\%) auf, wobei Nekrosen $(n=2,4 \%)$, chronische Diarrhö $(n=2,4 \%)$ und Stuhlinkontinenz $(n=7,13 \%)$ beobachtet wurden. 
Diskussion: Diese konsekutive Kohorte selektierter PatientInnen mit Analkanalkarzinom, die einer Brachytherapie zugänglich waren, zeigt nach Radiochemotherapie gefolgt von bildgestützter PDR-BT ein günstiges Ergebnis im Vergleich mit der Literatur.

\section{VAR-06}

\section{Navelbine bei der Trimodalen Therapie des inoperablen Harnblasenkarzinoms - Retrospektive Single-Center Auswertung der letzten 10 Jahre}

C. Arnold ${ }^{1}$, A. Lindner ${ }^{2}$, J. Mangesius ${ }^{1}$, D. Vasiljevic ${ }^{1}$, O. Kovaiou ${ }^{1}$, P. Lukas ${ }^{1}$, W. Horninger ${ }^{2}$, U. Ganswindt ${ }^{1}$, R. Pichler ${ }^{2}$, S. Skvortsov ${ }^{1}$

${ }^{1}$ Universitätsklinik für Strahlentherapie-Radioonkologie, Medizinische Universität Innsbruck, Innsbruck, Österreich ${ }^{2}$ Univ.-Klinik für Urologie, Medizinische Universität Innsbruck, Innsbruck, Österreich

Einleitung: Die organerhaltende trimodale Therapie (TMT) mit Cisplatin ist eine akzeptierte Alternative zur radikalen Zystektomie bei lokal begrenztem muskelinvasivem Harnblasenkarzinom (MIBC). Es gibt jedoch einen beträchtlichen Anteil an Patienten die Cisplatin nicht bekommen können und für die optimale Therapie dieses Patientenkollektivs gibt es nur unzureichende Daten. Wir berichten in dieser retrospektiven Single-Center Analyse über die onkologischen Ergebnisse von Patienten, bei denen im Rahmen der organerhaltenden Therapie eine Radiochemotherapie mit Cisplatin durchgeführt wurde, im Vergleich zu Patienten die ausschließlich bestrahlt wurden oder als Radiosensitizer Vinorelbin bekommen haben.

Methodik: In den vergangenen 10 Jahren (2008-2017) wurden 57 Patienten (m:w =45.12) an unserer Abteilung im Rahmen einer TMT bei MIBC behandelt. Vor Einleitung der Radio(chemo)therapie wurde zeitnah eine transurethrale Resektion des Blasentumors (TURB) durchgeführt. 12 Wochen nach Ende der Therapie und in weiterer Folge alle 3 und später alle 6 Monate wurden Kontrollzystoskopien und Re-Staging-CTs durchgeführt.

Ergebnisse: Das mediane Alter bei Beginn der Therapie betrug 80 Jahre (Range 48-91). 30 Patienten (52,6\%) erhielten Navelbine (RTnav), 12 Patienten $(21,1 \%)$ bekamen Cisplatin (RTcis) und 15 Patienten $(26,3 \%)$ wurden nur bestrahlt (RTonly). 54 Patienten $(94,7 \%)$ erhielten die geplante Dosis von 59,4 Gy (Lymphabflusswege 50,4 Gy, Harnblase 59,4 Gy). Das mediane Überleben betrug 34 Monate (RTcis), 22 Monate (RTnav) und 8 Monate (RTonly). Ein Lokalrezidiv bekamen $33 \%$ (RTcis), $37 \%$ (RTnav) und $47 \%$ (RTonly) der Patienten. Die mediane lokale Kontrolle war 38 Monate (RTnav), 37 Monate (RTcis) und 19 Monate (RTonly).

Diskussion: Die Gabe von Navelbine als Radiosensitizer im Rahmen der organerhaltenden TMT bei MIBC bei Patienten die nicht Cisplatintauglich sind führt zu einer fast Verdreifachung des medianen Überlebens und Verdoppelung der medianen lokalen Kontrolle auf CisplatinNiveau bei ausgesprochen guter Verträglichkeit und stellt somit eine gute Alternative für dieses Patientenkollektiv dar.

\section{VAR-07}

\section{Radiotherapie des Rektumkarzinoms im Spiegel der Zeit Erfahrungen am Landeskrankenhaus Feldkirch 1985-2014}

\section{H. Eiter, A. De Vries}

Abteilung für Radio-Onkologie, Landeskrankenhaus Feldkirch, Feldkirch, Österreich

Einleitung: Erstens, die Entwicklung der Strahlentherapie in der Behandlung des Rektumkarzinoms in den letzten 30 ahren aufzuzeigen und zweitens die erreichten Ergebnisse vorzustellen.
Dies speziell auch unter der Fragestellung ob die Umstellung der Therapie auf neoadjuvante Therapie einen Einfluss auf das Gesamtüberleben hat

Methodik: Die Akten aller Patienten mit der Diagnose Rektumkarzinom von 01.01.1985-31.12.2014 wurden herausgesucht und die Daten durch Nachfrage im zuweisenden Krankenhaus, beim Hausarzt und bei den Patienten selbst aktualisiert.

Im besagten Zeitraum wurden insgesamt 669 Patienten zugewiesen, davon wurden 260 PatientInnen (39\%) präoperativ, $159(24 \%)$ adjuvant,49 (7,3\%) nicht und $135(20,2 \%)$ in palliativer Intention behandelt

Ergebnisse: Die Zuweisung zur Radiotherapie änderte sich in Beobachtungszeitraum. In den 80igern und frühen 90iger Jahren diente die Strahlentherapie oftmals als ultima ratio, wenn alle anderen Möglichkeiten ausgeschöpft waren. Machte 1990-1994 die Bestrahlung eines Lokalrezidives noch $36 \%$ der Zuweisungen aus, gab es 2010-2014 keine Zuweisung für diese Indikation. Eine adjuvante Therapie führten wir zuletzt nur noch in ca. $5 \%$ der Fälle durch 1990-2000 jedoch in 30-40\%. Gegenläufig war die präoperative Radiochemotherapie. In den Jahren 1995-1999 erhielten $20 \%$ der Patienten mit Rektumkarzinom eine neoadjuvante Radiochemotherapie, 2010-2015 waren es $63 \%$.

Im Vergleich präoperative versus adjuvante Radiotherapie zeigt sich im Gesamtüberleben ein signifikanter Unterschied (5yOS $75 \%$, 10 yOS $53 \%$, 15yOS $41 \%$ versus 5 yOS $66 \%$, 10yOS $41 \%, 15 y$ OS $26 \%$ ) zu Gunsten der präoperativen Therapie. Auch in Hinblick auf die Lokalrezidivrate zeigt sich ein signifikanter Unterschied (8.9\% versus $23.5 \%$ ), wobei hier mit hoher Wahrscheinlichkeit die Einführung neuer Operationstechniken (Heald) den entscheidenden Vorteil erbrachte Diskussion: Bei der Radiotherapie des Rektumkarzinoms ist in den vergangenen Jahrzehnten ein bedeutender Wandel eingetreten, den wir auch anhand unseres Krankengutes beobachten konnten. Machten anfangs palliative Indikationen einen wesentlichen Teil der behandelten Patienten aus, ist mittlerweile die Radiotherapie ein wesentlicher Bestandteil eines kurativen Behandlungskonzeptes. Nicht nur die lokale Kontrolle sondern auch das Gesamtüberleben konnte bedingt auch durch den Wechsel auf ein präoperatives Vorgehen deutlich verbessert werden.

Dabei hat auch der Stellenwert des Faches eine deutliche Aufwertung erfahren.

\section{VAR-08}

\section{Neoadjuvante hyperfraktionierte akzelerierte Radiotherpaie des lokal fortgeschritten Rektumkarzioms}

J. Feichtinger, H. Geinitz, L. Kocik, C. Track, J. Kaufmann, T. Weingartner

Abteilung für Radio-Onkologie, Ordensklinikum Linz Barmherzige Schwestern, Linz, Österreich

Einleitung: Die neoadjuvante Kurzzeitbestrahlung $(5 \times 5 \mathrm{~Gy})$, alternativ Radiochemotherapie ist beim lokal fortgeschrittenen tief oder im mittleren Rektumdrittel lokalisierten Rektumkarzinom Therapiestandard. Spätnebenwirkungen (low anterior resektion syndrome - LARS) finden sich bei bis zu $50 \%$ der Stoma freien Patienten nach Kurzeitbestrahlung und tiefer anteriorer Resektion. Widder et al. publizierte 2005 eine alternative Fraktionierung mit $2 \times$ täglich 2,5 Gy und einer Gesamtdosis von 25 Gy in 10 Fraktionen.

Methodik: Im Zeitraum 2002-2017 wurden an unserer Abteilung 308 Patienten mit lokal fortgeschrittenem Rektumkarzinom UICC II - III neoadjuvant hyperfraktioniert akzeleriert behandelt. Die Radiotherapie wurde mit konventioneller Technik (pa/seitlich) in Bauchlage durchgeführt. Die Fraktionierung erfolgte mit $10 \times 2,5$ Gy in 5 Tagen. Zwischen den Fraktionen wurden 6 Stunden Pause angestrebt. Die Tumor- 
resektion war für die darauffolgende Woche vorgesehen. Primäres Ziel der retrospektiven Auswertung war die lokale Kontrolle (LC), das Gesamtüberleben (OS) sowie das krankheitsfreie Überleben (DFS). Die Akutnebenwirkungen, OP Komplikationen, Stomarate wurden erfasst. Spätnebenwirkungen (LARS Score) werden erhoben.

Ergebnisse: Das mittlere Alter der operierten Patienten betrug 69 (2794) Jahre. Die mediane Nachbeobachtung war 77 Monate. Lokalrezidive traten bei 11 Patienten (3,6\%) im Median nach 28,3 (8-92) Monaten auf. Das Gesamtüberleben nach 5 Jahren und 10 Jahren betrug $74,1 \%$ und $57 \%$, das krankheitsfreie Überleben nach 5 Jahren bzw. 10 Jahren $62,1 \%$ und 50,1\%. Es wurden keine Akutnebenwirkungen $>$ Grad 1 während der Radiotherapie beobachtet. Im Median betrug die Zeit nach Abschluss der Radiotherapie bis zur OP 5 (3-41) Tage. 273 Patienten (88,6 \%) wurden primär mit Stoma versorgt, das bei 112 Patienten im Median nach 183 Tagen rückoperiert werden konnte. Bei 45 Patienten $(14,6 \%)$ kam es in den ersten 30 postoperativen Tagen zu Komplikationen (Serom, Wundheilungsstörungen, Ileus ...).

Diskussion: Die neoadjuvante hyperfraktionierte akzelerierte Radiotherapie beim Rektumkarzinom ist eine gut verträgliche Therapieoption mit hoher lokaler Kontrollrate. Die kurze Gesamtbehandlungszeit bis zur Tumorresektion ist für die Patienten psychologisch ein zusätzlicher Vorteil.

\section{VAR-09}

\section{Endometriumkarzinom nach Hysterektomie: Bildgestützte Radiotherapie pelviner Rezidive}

J. Knoth ${ }^{1}$, D. Berger ${ }^{1}$, R. Pötter ${ }^{1}$, M. Schmid ${ }^{1}$, C. Grimm ${ }^{2}$, V. Seebacher ${ }^{2}$, S. Polterauer ${ }^{2}$, J. Widder ${ }^{1}$, A. Reinthaller ${ }^{2}$, A. Sturdza ${ }^{1}$

${ }^{1}$ Universitätsklinik für Strahlentherapie, Medizinische Universität Wien, Wien, Österreich

${ }^{2}$ Universitätsklinik für Gynäkologie, Medizinische Universität Wien, Wien, Österreich

Einleitung: Evaluierung von Tumoransprechen (TR), lokaler Kontrolle (LC), progressionsfreiem Überleben (PFS), Gesamtüberleben (OS) und Toxizität (TOX) nach Behandlung rezidivierter (Rez.) Endometriumkarzinome (EC) mit bildgestützter adaptiver Brachytherapie $($ IGABT) + /- Teletherapie (EBRT).

Methodik: Retrospektive Analyse aller aufgrund rez. EC zwischen 2001-2016 im AKH Wien behandelten Patientinnen, welche initial hysterektomiert (HE) wurden. Die Behandlung bestand aus IGABT bei auf die Vagina (V) begrenzten Tumoren (T) und N0-Status (Gruppe 1). Patientinnen mit die $\mathrm{V}$ überschreitenden $\mathrm{T}$ und/oder regionalen Lymphknoten-Metastasen (LK-SBL) erhielten eine EBRT + IGABT (Gruppe 2). Die IGABT-Planung erfolgte mit MRT (70\%) bzw. CT (30\%). Der T wurde als high risk-CTV, die V als low risk-CTV konturiert. Zieldosis für die V war $60 \mathrm{~Gy}$, für den T 85 Gy. LC, PFS und OS wurden mittels der Kaplan-Meier Methode berechnet, die Toxizität wurde mittels der CTCAEv4 Kriterien erfasst.

Ergebnisse: Das mediane Alter der Kohorte $(n=52)$ war 76 (53-91) Jahre (y), die mediane Nachbeobachtungszeit (FU) nach Behandlung des Rez. betrug 49 (2-184) Monate (m). Alle Patientinnen hatten initial ein Typ I EC, während 13 (25 \%) ein Typ II Rez. entwickelten. Die mediane Zeit von initialer HE bis Rez. betrug 26 m (5-130). 11 Patientinnen erhielten initial eine EBRT $(n=5)$ oder BT $(n=6)$. TR war $95 \%$; LC, PFS und OS nach 2/5y waren $95 \% / 95 \%, 74 \% / 69 \%$ sowie $85 \% / 78 \%$. 4 Pat. (2 vorbestrahlt) entwickelten urogenitale TOX Grad $\geq 3$ (vesicovaginale Fistel, Stenose) und/oder rectovaginale Fistel.

In Gruppe $1(n=9)$ hatte eine Patientin eine vorherige EBRT. Eine komplette Remission wurde immer erreicht, eine Patientin entwickelte ein LK-Rez. LC, PFS und OS nach 2/5y waren $100 \% / 100 \%$, $100 \% / 83 \%$ sowie $100 \% / 100 \%$. Es trat keine Grad $\geq 3$ TOX auf.
In Gruppe $2(n=43)$ hatten zwei Patientinnen eine inkomplette TR und entwickelten innerhalb von $3 \mathrm{~m}$ Lungen-SBL. 12/41 verbliebenen Pat. entwickelten SBL. LC, PFS und OS nach 2/5y waren $94 \% / 94 \%$, $69 \% / 66 \%$ und $82 \% / 74 \%$. Alle Grad $\geq 3$ TOX traten in dieser Gruppe auf (4/43 Patientinnen).

Diskussion: Lokoregionale Rezidive von initial operierten EC können mit Hilfe moderner strahlentherapeutischer Techniken, vor allem der IGABT, erfolgreich behandelt werden. Vorherige RT schließt eine Behandlung nicht aus. Bei auf die Vagina begrenzten Rezidiven ist die IGABT die Therapie der Wahl, bei die Vagina überschreitenden Rezidiven erscheint eine Kombination mit EBRT sinnvoll.

\section{VAR-10}

\section{Veränderungen der Neurokognition bei Protonentherapie im Schädelbereich}

P. Georg, L. Konrath, N. Gruber, A. Schallerbauer-Peter, A. Perpar, C. Lütgendorf-Caucig, R. Konstantinovic, C. Fussl, U. Mock, E.B. Hug

MedAustron Ionentherapiezentrum, Wiener Neustadt, Österreich

Einleitung: Patienten mit Tumoren im Schädelbereich, die mit Strahlentherapie behandelt werden, können neurokognitive Toxizität entwickeln. Die Protonentherapie bietet im Vergleich zu konventioneller Strahlentherapie das vorteilhafte invertierte Dosisprofil mit steilen Randabfällen und geringen Niedrigdosisvolumina. Um den klinischen Vorteil der Dosisreduktion im normalen Gewebe zu prüfen, haben wir die neurokognitive Funktion vor und nach einer Protonentherapie im Schädelbereich getestet.

Methodik: Es wurde eine standardisierte EORTC Testbatterie verwendet. Der Hopkins Verbal Learning Test (HLTV-R) testet sofortiges und verzögertes verbales Gedächtnis. Trail Making Test Part A und B (TMT-A und B) beurteilt Aufmerksamkeit und Verarbeitungsgeschwindigkeit sowie kognitive Flexibilität. The Controlled Oral Word Association Test (COWA) wird zur Beurteilung der Wortflüssigkeit und exekutiven Funktion herangezogen. Der Grooved Pegboard Test evaluiert motorische Koordination. Die evaluierten Testzeitpunkte waren vor Therapiebeginn, am Ende der Therapie, 3 und 6 Monate nach Therapieende. Weitere Messpunkte sind $1 \times$ jährlich über den Zeitverlauf von 10 Jahren (Daten liegen noch nicht vor).

Ergebnisse: Die ersten 4 Messzeitpunkte (Baseline, Therapieende, 3 und 6 Monate nach Therapieende) wurden bei 112, 94, 61 und $40 \mathrm{~Pa}$ tienten durchgeführt. Keine signifikanten Veränderungen waren bislang erkennbar bei Verbalem Gedächtnis (HVLT-R), kognitiver Flexibilität, exekutiver Funktion (TMT-B) und motorischer Koordination (Grooved Pegboard). Es kam zur signifikanten Verschlechterung von Verarbeitungsgeschwindigkeit (TMT-A, $p=0,002$ ), von mittlerem Wert $32,8 \pm 1,4$ vor Therapiebeginn auf 26,8 $\pm 1,4$ nach 6 Monaten. Es kam zur signifikanten Verbesserung bei Wortflüssigkeit (COWA, $p=0,000$ ) von mittlerem Wert $28,1 \pm 8,4$ vor Therapiebeginn auf $36,7 \pm 8,4$ nach 6 Monaten.

Diskussion: Erstmalig wurden prospektiv wiederholte neurokognitive Tests in einem großen Kollektiv von Patienten, die mit Protonentherapie im Schädelbereich behandelt wurden, durchgeführt. Die Tests wurden von den Patienten als zumutbar akzeptiert und die Compliance war sehr hoch. Initiale Ergebnisse bestätigen, dass sich neurokognitive Teilbereiche unterschiedlich verändern können. Weitere Analysen mit längerem Follow-up und Subgruppenanalysen, sowie Korrelation mit klinischen Outcome und Bestrahlungsdaten sind geplant. 


\section{VAR-11}

\section{Rebestrahlung mit Protonen - der erste Bericht nach 6 Monaten Follow-up}

A. Perpar, P. Georg, C. Fussl, C. Lütgendorf-Caucig, U. Mock, L. Exeli, R. Konstantinovic, G. Kragl, M. Stock, E. B. Hug

MedAustron Ionentherapiezentrum, Wiener Neustadt, Österreich

Einleitung: Aufgrund der günstigeren Dosisverteilung ermöglicht die Protonentherapie eine bessere Schonung der gesunden Gewebe in der Umgebung der Zielvolumina. Bei radiogener Vorbelastung ist diese Eigenschaft von besonderer Bedeutung. Das Risiko für schwerwiegende therapiebedingte Toxizitäten kann durch eine Protonenbestrahlung bei zeitgleicher Applikation einer kurativen Dosis reduziert werden.

Methodik: Die Patientenselektion erfolgte auf Basis von festgelegten Kriterien, wie z.B. Lokalrezidiv ohne distante Metastasierung sowie gute Toleranz der Erstbestrahlung. Die Dosisvorschreibung erfolgte bis zu einer kurativen Dosis, in einem normo- oder moderat hypofraktionierten Schema; die Gesamtdosis wurde entsprechend der zugrunde liegenden Erkrankung festgelegt. Die therapiebedingten Nebenwirkungen wurden mittels CTCAE v.4.0 klassifiziert.

Ergebnisse: Zwischen 10/2017 und 08/2018 wurden insgesamt $21 \mathrm{~Pa}-$ tienten mittels Protonen-Rebestrahlung behandelt. Das mediane Alter lag bei 52,9 Jahren (Range 7-81), das mediane Zeitintervall zur Rebestrahlung betrug 44 Monate (Range 12-191). Die behandelten Lokalisationen waren im Bereich der Schädelbasis $(62 \%, n=13)$, des ZNS (5 \%,n=1), des Gesichtsschädels $(19 \%, n=4)$ sowie des Halses $(14 \%, n=3)$; Histopathologisch handelte es sich in $52 \%(n=11)$ um Plattenepithelkarzinom, in $24 \%(n=5)$ um Meningeome, in $9 \%(n=2)$ um Weichteilsarkome sowie in $15 \%(n=3)$ um andere Histologien. Die gewählte Gesamtdosis betrug zwischen 50,4 und 72 Gy RBE. Bei $86 \%(n=19)$ der Patienten traten ausschließlich geringgradige akute Nebenwirkungen (Grad 1-2) auf. Drei Patienten (14\%) entwickelten eine akute Grad-3-Nebenwirkung.

Nach Therapieabschluss und einer medianen Beobachtungszeit von 6 Monaten (Range 1-17) wurden bei 5 Patienten (23\%) Grad-3-Spättoxizitäten (4 Patienten Nekrosen, 1 Blutung) dokumentiert. 1 Patient ist 8 Monate nach Behandlungsabschluss unter der Annahme einer Progression innerhalb des Bestrahlungsfeldes verstorben.

Diskussion: Erste Behandlungsdaten aus unserem Zentrum belegen, dass eine Rebestrahlung mit Protonen mit akzeptablen frühen Nebenwirkungen verbunden ist.

\section{VAR-12}

\section{Monozenter-Ergebnisse hypofraktionierter extrakranieller stereotaktischer Strahlentherapie (SBRT) von Lungenmetastasen}

M. Nevinny-Stickel, B. Weiland, D. Vasiljevic, I. Kvitsaridze, C. Arnold, J. Mangesius, P. Lukas, T. Seppi, U. Ganswindt

Universitätsklinik für Strahlentherapie-Radioonkologie, Medizinsche Universität Innsbruck, Innsbruck, Österreich

Einleitung: Im Zeitraum von 01/2012 bis 12/2017 wurden 161 Lungenmetastasen von 96 Patienten mittels SBRT behandelt. Lokalkontrolle und Gesamtüberleben werden vorgestellt.

Methodik: 161 Lungenmetastasen von 96 Patient (28 Kolorektalkarzinome, 26 HNO-Tumore, 13 gynäkologische Tumore, 11 Hauttumore, 10 Urogenitaltumore und 8 andere Primärtumore) wurden mit einer medianen Gesamtdosis von 45 Gy (65\%-Isodose, Range: $42-60$ Gy), sowie einer medianen Einzeldosis von 15 Gy (Range: 6-15 Gy) bestrahlt. Die SBRT wurde mit 3 bis 10 Fraktionen (Median 3) und median 6 (Range: 3-10)Bestrahlungsfeldern durchgeführt. Medianes Alter der Patienten war 69 a (Range: 16-90). 60 \% der Patienten hat- ten eine, $23 \%$ zwei, $12 \%$ drei und 5\% 4-6 Metastasen. Das PTV betrug im Median 14,2 ccm (Range: 1.5 bis $87 \mathrm{ccm}$ ). Die Nachsorge mittels CT erfolgte im ersten Jahr alle 3 Monate, ab dem zweiten Jahr alle 6 Monate.

Ergebnisse: Im medianen Nachbeobachtungszeitraum von 13 Monaten (Range: 1-63 Monate) beträgt die lokale Kontrollrate 96,9\%. Das Gesamtüberleben im Beobachtungszeitrum beträgt $73 \%$. Bei $52 \%$ der Patienten traten nach SBRT neue weitere Lungenmetastasen auf, die teilweise wieder mit SBRT behandelt wurden. Grad IV Nebenwirkung traten nicht auf, 9 Patienten bekamen transiente Grad II-III Pneumonitiden. Bisher sind 26 Patienten verstorben (5 gesichert am Tumorprogress). 85 von 96 Patienten befinden sich noch in der Nachbeobachtung.

Diskussion: Die SBRT ist wegen der geringen Nebenwirkungsrate und hohen lokalen Kontrollrate eine sehr gute Behandlungsoption von Lungenmetastasen, die auch bei mehreren Lungenmetastasen angewandt werden kann.

\section{RT-Programm}

RTP-01

\section{S\&S versus VMAT}

T. Deisenhammer

Universitätsklinik für Radioonkologie und Radiotherapie, Uniklinikum Salzburg-Landeskrankenhaus, Salzburg, Österreich

Einleitung: Unsere Erfahrungen mit beiden Techniken im Vergleich Methodik: Vergleich anhand verschiedenen Entitäten

Ergebnisse: Es gibt keine eindeutige Aussage, welche Technik zu bevorzugen ist.

Diskussion: Jeder Plan ist individuell zu beurteilen. Welche Technik bei der Bestrahlung angewendet wird hängt von mehreren Faktoren ab, die schlussendlich der Arzt mit Unterstützung von Planung und Physik abwägen muss.

\section{RTP-02}

Video-assistierte- Ruhigstellung (VAR) von Kindern und Jugendlichen während der teletherapeutischen Bestrahlung

N. Ritt, S. Hofstätter, A. Osztavics, D. Berger, B. Wisgrill, K. Dieckmann

Universitätsklinik für Strahlentherapie, Medizinische Universität Wien, Wien, Österreich

Einleitung: Um tägliche Sedierungen von Kindern bei TeletherapieBestrahlungen zu verringern, wurde ein Pilotprojekt von Juli 2017Mai 2018 an der Universitätsklinik für Strahlentherapie am Allgemeinen Krankenhaus Wien (UKSTR) gestartet. Mittels Kurzfilme auf Mini-Tablets, sollen die Kinder kognitiv abgelenkt werden um die Position am Behandlungstisch während der Bestrahlung einzuhalten und gleichzeitig Stress- und Angstzustände zu vermeiden bzw. zu verringern.

Darüber hinaus sollte die Anzahl der gleichzeitig täglichen radioonkologischen Behandlungen bei Kindern und Jugendlichen an der UKSTR gesteigert werden. Dadurch wäre der ökonomische Aspekt welcher die Kostenreduzierung des optimalen Einsatzes der vorhandenen Ressourcen erfüllt.

Methodik: Basierend auf ärztlichen Vorgaben der Durchführbarkeit, wird primär allen PatientInnen (0-18 Jahren) die Möglichkeit der VAR- Nutzung seitens der RadiologietechnologInnen (RT's) angebo- 
ten. Mittels standardisierter Evaluierung werden die individuellen Bedürfnisse in den VAR- Ablauf implementiert. Um den optimalen Output der VAR- Methodik erreichen zu können ist es unabdingbar, dass zum Zeitpunkt der radioonkologischen Therapievorbereitung (Planungs- CT, Simulation) die Kinder und Jugendlichen bereits kognitiv abgelenkt werden. Die adäquate Vorbereitung liegt im Zentrum des Pilotprojektes.

Ergebnisse: In diesem Zeitraum (Juli 2017-Mai 2018) wurden insgesamt 38 Kinder und Jugendliche, davon 24 männlich und 14 weiblich, im Alter von 0-18 Jahren behandelt. $52 \%$ der behandelten Kinder und Jugendlichen wurden mittels Filmen kognitiv während der Bestrahlung abgelenkt. $34 \%$ wollten während der Bestrahlung keinen Film sehen und $13 \%$ mussten für Bestrahlung sediert werden.

Wir konnten punktuell eine Steigerung von $50 \%$ an der UKSTR erzielen.

In diesem Zeitraum (Juli 2017-Mai 2018) wurden insgesamt 38 Kinder und Jugendliche, davon 24 männlich und 14 weiblich, im Alter von 0-18 Jahren behandelt. $52 \%$ der behandelten Kinder und Jugendlichen wurden mittels Filmen kognitiv während der Bestrahlung abgelenkt. $34 \%$ wollten während der Bestrahlung keinen Film sehen und $13 \%$ mussten für Bestrahlung sediert werden.

Wir konnten punktuell eine Steigerung von $50 \%$ an der UKSTR erzielen.

Diskussion: Es zeigt sich, dass die VAR- Methode optimal in den klinischen Betrieb implementiert werden kann und die gesetzten Ziele dieses Projektes erreicht wurden. Wesentliche Voraussetzung zum Erfolg dieser Methode ist das gezielte Briefing des Kindes und deren Begleitperson, sowie aller beteiligten Personen der jeweiligen Berufsgruppen (RT's Pflege, ÄrztInnen).

Ein nicht zu vernachlässigender Nebenaspekt ist, eine Kostenreduktion pro Behandlungsserie und Kind von ca. $€ 2800$, sowie eine effiziente Nutzung der bestehenden Ressourcen (Personal-, Gerätebindungszeit) durch die klinische Nutzung der VAR- Methode, an der UKSTR.

\section{Physikprogramm}

\section{PHY-01}

\section{On the road towards MRI guided particle therapy: technological challenges and clinical potential}

H. Fuchs ${ }^{1,2}$, F. Padilla-Cabal ${ }^{1,2}$, L. Fetty ${ }^{1,2}$, P. Kuess ${ }^{1,2}$, T. Schreiner ${ }^{3}$, D. Georg ${ }^{1,2}$

${ }^{1}$ Department of Radiotherapy, Medical University of Vienna, Vienna, Austria

${ }^{2}$ Christian Doppler Laboratory for Medical Radiation Research for

Radiation Oncology, Vienna, Austria

${ }^{3}$ MedAustron centre for ion therapy, Vienna, Austria

Introduction: Particle therapy (PT) has become an optimal choice for tumors where conventional radiotherapy options are limited. However, dose delivery accuracy is by far more sensitive to anatomical changes and inter- and intrafraction movements. Magnetic resonance imaging (MRI) is a promising candidate for real-time image guidance during PT due to the superior soft tissue contrast, lack of exposure with ionizing radiation and the combination of anatomic and functional imaging. The clinical implementation of MRI guided X-ray beam therapy (MRXT) is ongoing with first MR-linacs ready for routine use. These developments have motivated our proof of principle research toward MR guided proton therapy.

Methods: Technical and physical challenges that need to be overcome are among others: interactions between the scanner and the beam line, image quality during irradiation, treatment planning based on MRI images only and dosimetric changes induced by the treatment beams deflection within the MR scanner. At the Medical University of Vienna, a dedicated research project aims to study key concepts for MR guided ion-beam therapy, with a special focus on pancreatic cancer and cranial lesions as use cases for MR guided proton therapy.

Results: Methodologies and algorithms are being developed for treatment planning and dose calculations for MRPT. Results comparable to existing systems had been achieved for the generation of synthetic CTs derived from MRI data sets using a neural network architecture. The peak signal noise ratio and the mean absolute error of the whole body between synthetic $\mathrm{CT}$ and planning $\mathrm{CT}$ was $31.57+-0.87 \mathrm{~dB}$ and $64.71+-7.79 \mathrm{HU}$, respectively. Also, a fast semi-analytical algorithm for ion beams in the presence of magnetic fields was developed. Comparison with Monte Carlo simulations using the Gate/Geant4 toolkit showed very good agreement between the simulated values and the algorithms predictions. Tests performed on calibration phantoms showed a maximal deviation on range prediction of $0.7 \mathrm{~mm}$, lateral beam deflection of $0.9 \mathrm{~mm}$ and gamma indexes $(2 \%, 2 \mathrm{~mm})$ pass rates higher than 98.5 for bidimensional dose distributions. Using a dedicated research magnet, experimental measurements and further validation in a proton beamline at the ion therapy center MedAustron in Wiener Neustadt are currently underway.

Conclusion: Although there are still open questions to be tackled, MRPT is a promising candidate to harvest the full potential of particle therapy and potentially extend the current clinical applications.

\section{PHY-02}

\section{Intensitätsmodulierte Protonen- und Photonentechniken mit simultan integriertem Boost in der Behandlung von Gliomen - gibt es einen relevanten Unterschied?}

G. Kragl1 ${ }^{1}$, J. Reiterer ${ }^{2}$, C. Lütgendorf-Caucig ${ }^{1}$, C. Steffal ${ }^{2}$, J. Gora, T. Kann, M. Stock ${ }^{1}$, A. U. Schratter-Sehn' ${ }^{2}$ E. B. Hug ${ }^{1}$

${ }^{1}$ MedAustron Ionentherapiezentrum, Wiener Neustadt, Österreich ${ }^{2}$ Institut für Radioonkologie, Sozialmedizinsches Zentzrum SüdKaiser Franz Josef Spital, Wien, Österreich

Einleitung: Nachteile der Strahlentherapie im multimodalen Behandlungskonzept von Gliomen sind therapiebedingte Toxizität wie zerebrovaskuläre und kognitive Veränderungen. Intensitätsmodulierte Techniken mit Simultan Integriertem Boost (SIB) zeigen einen Vorteil hinsichtlich der Schonung des Normalgewebes. Ziel der vorliegenden Untersuchung war es, relevante Unterschiede zwischen einer Protonen- und einer Photonentechnik zu identifizieren.

Methodik: Es wurden Planungsvergleiche intensitätsmodulierter Techniken mit SIB für Protonen und Photonen (VMAT) bei 3 WHO II und 4 WHO III Gliomen erstellt. Das CTV1 entsprach dem Gliom und einem $<15$ mm Margin im Kompartiment. Das CTV2 (Boost) beinhaltete nur das Gliom. Der PTV Margin betrug je $3 \mathrm{~mm}$. Für WHO II Gliome wurde 50,4 Gy für das PTV1 und 54,0 Gy für das PTV2 vorgeschrieben, für WHO III Gliomen 54,0 Gy für das PTV1 und 60,0 Gy für das PTV2 in 1,8 Gy-2 Gy Einzeldosis. Für Protonen wurde ein RBE von 1,1 angewendet. Für beide Modalitäten galten dieselben Planungsvorgaben für Targets und Toleranzdosen für Risikostrukturen (OAR). Die Planung erfolgte ICRU konform.

Ergebnisse: Das mediane PTV1 war 269,8 cm3 (Range 173,5-478,7), das mediane PTV2 154,1 cm3 (Range 70,7-191,5). Protonenplanung erfolgte mit energie-variiertem Pencil Beam Scanning. Die Optimierung erfolgte mittels Einzel-Feld-Optimierung. Photonenplanung erfolgte mittels VMAT-Technik. Die Zielvolumenabdeckung (Planungsvorgabe) und die Einhaltung der OAR Constraints waren für beide Modalitäten vergleichbar. Die mediane Dosis des Total Normal Tissue Volume brain $(\mathrm{TNTVb}=$ brain-PTV) betrug 1,5 Gy(RBE) (Range 0,2-9,5) für Protonen und 19,7 Gy (Range 16,7-26,0) für VMAT. Das mediane 10 
Gy TNTVb betrug 33,5\% (Range 23,4-49,4) mit Protonen und $76 \%$ (Range 57,1-89,5) mit VMAT. Das mediane 5 Gy TNTVb 40,8 \% (Range 27,3-56,6) mit Protonen und 83,3\% (Range 63,2-93,6) mit VMAT. Diskussion: SIB mit Protonen und VMAT zeigen gute PTV Abdeckung unter Einhaltung der OAR Toleranzdosen. Protonen haben darüber hinaus einen dosimetrischen Vorteil im Niedrigdosisbereich des ZNS. Neurokognitive Tests sind routinemäßig Teil der prospektiven Evaluation im MedAustron. Eine Korrelation von Protonendosisverteilung mit langzeitigem klinischen Outcome ist geplant.

\section{PHY-03}

\section{Vergleich von Log-File Analyse und Verifikationsmessung zur Qualitätssicherung von Intensitäts-modulierten Bestrahlungsplänen}

\section{Kowatsch, P. Szeverinski, M. Meinschad, T.Künzler}

Institut für Medizinische Physik, LKH Feldkirch, Feldkirch, Österreich

Einleitung: Eine Überprüfung der Datenübertragung von Bestrahlungsparametern ist durch die DIN 6875-3 vorgegeben. Dabei werden vier mögliche Verfahren vorgeschlagen. Die derzeit etablierteste Methode ist die ,dosimetrische Kontrolle jedes fluenzmodulierten Strahlenfeldes bzw. des Gesamtplans vor der ersten Bestrahlung“. Dies kann mit verschiedenen Messmethoden unter hohem Zeit- und Personalaufwand durchgeführt werden. In unserer Klinik wird die QA mit dem ArcCheck (SunNuclear) durchgeführt. Eine weitere Methode ist die „,nachträgliche Rekonstruktion der Teilchenfluenz-Verteilung und visueller oder automatisierter Vergleich mit den vom Bestrahlungsplanungssystem berechneten Teilchenfluenzen, falls die genauen Positionen aller Lamellen während der Bestrahlung gespeichert werden“. LinacWatch (Qualiformed) stellt diese Funktion zur Verfügung. In dieser Studie wird das Messsystem ArcCheck mit der Log-File Analyse LinacWatch verglichen.

Methodik: 20 verschiedene VMAT-Pläne verschiedenster Komplexität, die in der Therapie verwendet wurden und die den Qualitätskriterien des Instituts für Medizinische Physik am LKH Feldkirch entsprachen, wurden nochmals abgestrahlt und die aufgezeichneten Logdateien mit der Software LinacWatch analysiert. Dabei wurde die errechnete Fluenz anhand der Log-Dateien mit der errechneten Fluenz aus dem RTPlan File mit einem Gammakriterium von $2 \% / 2 \mathrm{~mm}$ und einem Schwellwert von $5 \%$ ausgewertet. Für die ArcCheck Messungen wurde ein Kriterium von $2 \% / 2 \mathrm{~mm}$ bei einem Schwellwert von $20 \%$ angewendet.

Ergebnisse: Die Ergebnisse zeigen, dass mit Linacwatch tendenziell höhere Passingrates erreicht werden als mit dem ArcCheck. So wurde für drei Hirnmetastasen mit dem ArcCheck eine Passingrate von $88.8 \%$, $84.1 \%$ und $87.9 \%$ erreicht, alle unterhalb der intern definierten Toleranzgrenze. Mit LinacWatch wurde eine Passingrate von $99.9 \%, 100 \%$ und $100 \%$ erzielt. Im Mittel lag die Passingrate für die 20 Pläne bei $94.1 \%$ für das ArcCheck und $98.9 \%$ für LinacWatch. Weiters sieht man, dass mit zunehmender Größe der Bestrahlungsvolumina die Passingraten zwischen den beiden Systemen stärker korrelieren.

Diskussion: Unter der Voraussetzung, dass alle Kalibrierungen am Linearbeschleuniger korrekt durchgeführt wurden, kann mit LinacWatch eine einfache und schnelle Planverifikation am Beschleuniger realisiert werden. Dies kann als Fraktion Null oder während der ersten Fraktion geschehen. Weiters bietet das System eine Überwachung aller Fraktionen an, dies ist mit einer Messung vor der Behandlung nicht möglich. Die höheren Passingraten beim LinacWatch erklären sich dadurch, dass Fehler im Dosisberechnungsalgroithmus nicht in die Analyse einfließen. Ein weiterer Grund liegt in der besseren Auflösung der Messdetektoren des Beschleunigers, die durch Linacwatch evaluiert werden. Je kleiner die Zielvolumina werden, umso mehr weichen die Ergebnisse beider Systeme voneinander ab.
Poster

POS-01

VMAT-technique (volumetric modulated arc therapy), IMRT-technique (intensity modulated radiotherapy) and 3D-conformal radiation for lymphomas in Radiation Oncology department in Bank of Cyprus Oncology Center

M. Theodorou ${ }^{1}$, A. Peratikou ${ }^{2}$, S.Stefanou ${ }^{2}$, L. Ioannou ${ }^{2}$, E. Themos ${ }^{2,}$ A. Artikan ${ }^{2}$, N. Poullos ${ }^{3}$, D. Andreopoulos ${ }^{1}$

${ }^{1}$ Department of Radiation Oncology, Banc of Cyprus Oncology Center, Nikosia, Cypros

${ }^{2}$ Medical Physics Department, Banc of Cypros Oncology Centre, Nikosia, Cypros

${ }^{3}$ Clinic for Radiooncology and Radiotherapy, Technical University Munich, Munich, Germany

Introduction: The purpose of this project was to evaluate 2 different treatment planning techniques, 3D conformal and Volumetric Modulated Arc Therapy (VMAT) for Non Hodgkin's Lymphoma and Hodgkin's Lymphoma.

Methods: All patients were planned on MONACO treatment planning system using both VMAT and 3D conformal techniques. The Radiation Oncologist delineated the CTVs/PTVs and Organs at Risk (OAR). 3D conformal and VMAT plans were performed and optimized by Medical physicists. Dose Volume Histograms (DVHs) for both modalities were analyzed by a statistician.

Results: Statistical analysis was carried out for the PTV coverage by $95 \%$ of the prescription dose for 3D conformal and VMAT plans. For the 3D conformal plans the mean coverage of the PTV by $95 \%$ of the prescription dose was $96.8 \%$ ( $\mathrm{SD}=2.69)$. For the VMAT plans the mean coverage of the PTV by $95 \%$ of the prescription dose was $98.67 \%$ $(\mathrm{SD}=0.95)$. Statistical analysis for the spinal cord as an OAR was carried out for 43 patients (in the remaining 17 patients the spinal cord was not in the vicinity of the PTV). It was found that the mean of the maximum absorbed dose (in Gy) by the spinal cord was less for VMAT plans i.e. $24 \mathrm{~Gy}$ (SD 9.4 Gy) compared to the 3D plans where the mean of the maximum absorbed dose was found to be $29 \mathrm{~Gy}$ (SD 12.2 Gy)

Conclusion: After comparison of individual patient plans, it was noted that for 48 out of 60 patients, the VMAT plans delivered higher coverage to the targets with $95 \%$ of the prescribed dose. For 43 patients the spinal cord sparing was evaluated as an OAR. In 31 of these patients the spinal cord sparing was better for the VMAT plans. In conclusion therefore, we suggest that the VMAT technique can be implemented for Radiotherapy of Lymphomas since it demonstrates superior PTV coverage and better OAR sparing, compared to 3D conformal methods.

\section{POS-02}

Strahlentherapiemanagement beim Seminom im klinischen Stadium I - eine retrospektive Analyse der Patienten im KFJ/SMZ-Süd Wien mit einem Nachbeobachtungszeitraum von über 20 Jahren

\section{Steffal, T. Kann, J. Reiterer, A. U. Schratter-Sehn}

Institut für Radioonkologie, Sozialmedizinsches Zentrum Süd-Kaiser Franz Josef Spital Wien, Wien, Österreich

Einleitung: Fast alle Patienten mit einem Seminom im klinischen Stadium I werden ungeachtet der adjuvant anschließenden Therapie geheilt. Adjuvante Chemotherapie, Surveillance und adjuvante Radiatio waren viele Jahrzehnte als Behandlungsoptionen bei Seminomen im klinischen Stadium I (CSI) allgemein akzeptiert; derzeit befinden sich 
fast alle Patienten in „Active Surveillance“. Wir führten eine Analyse bei allen im Institut ab 1997 adjuvant bestrahlten CSI Patienten durch. Methodik: In der Zeit von Jänner 1997 bis Februar 2018 wurden 82 Patienten mit einem Seminom im CSI einer postoperativen Radiotherapie unterzogen. Das mittlere Alter der Patienten betrug 37 Jahre (20-66a). Bei allen Patienten erfolgte eine Beurteilung der akuten Nebenwirkungen nach den CTCAE-Kriterien, der chronischen Nebenwirkungen nach LENT/SOMA-Kriterien, eine Evaluierung des Hormonstatus, der psycholgischen Situation, der Rezidivmuster und des Auftretens von Zweitmalignomen.

Ergebnisse: Das mediane Follow up der retrospektiven Analyse betrug 159 Monate $(60-258)$. Akute Nebenwirkungen nach CTCAE Grad 1 traten bei $47.37 \%$, nach CTCAE Grad 2 bei $19.74 \%$ der Patienten auf. Spätfolgen, klassifiziert nach LENT/SOMA-Kriterien, konnten bei $10.53 \%$ der Patienten evaluiert werden. $12 \%$ aller Patienten hatten pathologische Hormonwerte. Es gab kein retroperitoneales Rezidiv, an Zweitmalignomen wurde ein Melanom am Feldrand 11 Jahre nach RT beobachtet.

Es wurde literaturkonform eine mittlere Gesamtherddosis von 25.2 Gy (20-30.6 Gy) verabreicht. Das krankheitsfreie Überleben betrug nach 5 Jahren $100 \%$, nach 10 Jahren $97.4 \%$.

Diskussion: Diese retrospektive Analyse zeigt, dass die adjuvante retroperitoneale Radiotherapie bei Seminomen im klinischen Stadium I ein optimales Therapiemanagement ohne gravierenden Spätfolgen darstellt, wenn Patienten die „Active Surveillance“ ablehnen.

\section{POS-03}

\section{Postradiogene Morphea der Brust: Fallbericht und Literaturüberblick}

\section{R. Partl ${ }^{1}$, P. Regitnig ${ }^{2}$, E. Trapp ${ }^{3}$, K. S. Kapp ${ }^{1}$}

${ }^{1}$ Univ. Klin. f. Strahlentherapie-Radioonkologie, LKH-Univ.Klinikum Graz, Graz, Österreich

${ }^{2}$ Univ. Institut f. Pathologie, LKH-Univ.Klinikum Graz, Graz,

Österreich

${ }^{3}$ Univ. Klin. für Frauenheilkunde u. Geburtshilfe, LKH-Univ.

Klinikum Graz, Graz, Österreich

Einleitung: Die Morphea ist eine Autoimmunreaktion des subkutanen Bindegewebes, die unter anderem durch Radiotherapie getriggert wird. Sie wird am häufigsten nach adjuvanter Brustbestrahlung beobachtet. Methodik: 2017 wurde an unserer Klinik erstmals eine Morphea diagnostiziert. Die Diagnose erfolgte stanzbioptisch. Um eine zielgerichtete Therapie anbieten zu können wurde eine Literaturrecherche mit den Begriffen ,radiation-induced morphea, localized scleroderma, radiation port scleroderma und breast cancer" durchgeführt. 2018 wurde bei einer weiteren Patientin eine bilaterale Morphea nach Radiatio beider mammae verifiziert.

Ergebnisse: In den letzten 20 Jahren wurden 40 Fälle von postradiogener Morphea der Brust beschrieben. In der Literatur werden Inzidenzraten von 1:500 bis 1:3000 angeführt, die nicht mit der geringen Zahl an Fallberichten korrelieren. Nach einem beschwerdefreien Intervall von einem Monat bis mehreren Jahren treten die Symptome (umschriebene Entzündung, Ödem) häufig abrupt auf und sind klinisch von einem inflammatorischen Rezidiv oder einer Entzündung nicht zu unterscheiden. In der Spätphase ist die Morphea von einer radiogenen Fibrose abzugrenzen. Der auslösende Mechanismus, der zur Expression der Zytokine IL $4 \& 5$ und TGF- $\beta$ führt, Fibroblasten aktiviert und die Kollagensynthese steigert, ist unklar.

Diskussion: Neben Radiotherapie, Bindegewebstraumata und Infektionen könnten neoadjuvant verabreichte tumorwirksame Medika oder deren Kombination mit subsequenter Bestrahlung einen Trigger für die Entwicklung einer Morphea darstellen. Offenbar wird diese seltene schwere Komplikation im initialen Stadium als Infektion und im weiteren Verlauf als postradiogene Fibrose fehlinterpretiert und nicht oder falsch behandelt. Bei klinischer Verdachtsdiagnose und zum Ausschluss eines Lokalrezidivs muss die Diagnose stanzbioptisch verifiziert werden. Die frühzeitige Behandlung mit systemischer und lokaler Immunsuppression (Methotrexat, Kortikosteroide, UVA/UVB) im inflammatorischen Stadium kann das Risiko für eine irreversible Fibrose und Atrophie der Brust reduzieren und die Lebensqualität verbessern. Eine operative Sanierung scheint nicht zielführend.

\section{POS-04}

\section{Thalidomid mildert frühe Strahlenzystitis bei Mäusen}

\section{J. Kowaliuk ${ }^{1}$, P. Kuess ${ }^{1}$, W. Dörr ${ }^{1,2}$}

${ }^{1}$ Universitätsklinik für Strahlentherapie, Medizinische Universität Wien, Wien, Österreich

${ }^{2}$ Christian Doppler Labor für Medizinische Strahlenforschung für die Radioonkologie, Wien, Österreich

Einleitung: Die Strahlentherapie ist eine effektive Methode zur Behandlung solider Tumoren im Becken, welche Prostata-, Harnblasen-, Rektal-, und Zervixtumore beinhalten. Im Laufe dieser Behandlung wird die Harnblase häufig Dosen ausgesetzt, welche zu Nebenwirkungen führen können und als Strahlenzystitis bekannt sind. Die Symptome basieren auf einer Reduktion des Blasenvolumens und manifestieren sich anhand einer Erhöhung der Miktionsfrequenz, Dysurie, Inkontinenz und Nykturie. Die Symptome erfolgt in drei verschiedenen Phasen: (1) eine reversible biphasische akute Reaktion, (2) eine symptomfreie Phase von Monaten bis Jahren und (3) eine irreversible Spätphase. Studien zeigten, dass frühe strahlen-induzierte Entzündungsprozesse an der Pathogenese beteiligt sind. Daher wird Thalido-

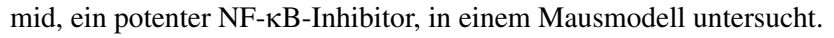
Methodik: Diese präklinische Studie wurde in einem gut etablierten Mausmodell in weiblichen $\mathrm{CH} 3 / \mathrm{Neu}$ Mäusen durchgeführt. Die Bestrahlung erfolgte unter Anästhesie, wobei die Blasen in einem rechteckigen Expositionsfeld unter Verwendung von abgestuften Einzeldosen bestrahlt wurden. Der Darm wurde mittels Kummerbundtechnik aus dem Bestrahlungsfeld entfernt. Das Blasenvolumen wurde mittels transurethraler Zystotonometrie in 3-Tages-Intervallen in der ersten Phase (Tag 0 bis Tag 30) bestimmt. Die Endpunkte wurden als $>50 \%$ Verringerung des ursprünglichen Blasenvolumens bei $10 \mathrm{mmHg}$ intravesikulärem Druck festgelegt. Gemäß der biphasischen frühen und späten Phase (Tag 1-15 und 15-30) wurde Thalidomid systemisch täglich (100 mg/ $\mathrm{kg}$ intraperitoneal) über verschiedene Zeitintervalle verabreicht.

Ergebnisse: Die tägliche Anwendung von Thalidomid von Tag 1 bis Tag 15 erhöhte die Strahlenverträglichkeit signifikant von EC50 $=15,5 \pm 8,8$ Gy auf 55,4 $\pm 24,0$ Gy in Phase 1 (Tag 1-15) und von $\mathrm{EC} 50=13,7 \pm 4,2$ Gy bis $23,1 \pm 4,2$ Gy in Phase 2 (Tag 15-30). Die tägliche Applikation von Tag 15-30. erhöhte die Strahlentoleranz von EC50 $=13,7 \pm 4,2$ Gy auf $32 \pm 28,0$ Gy in Phase 2 (Tag 15-30).

Diskussion: Die Daten zeigen dass Thalidomid ein potentielles Medikament zur Behandlung und Linderung der frühen Strahlenzystitis ist und womöglich Einfluss auf die Bildung von Spätreaktionen hat.

\section{POS-05}

Bedarf und Wunsch nach psychoonkologischer Behandlung zu Beginn der ambulanten Strahlentherapie - Ergebnisse eines psychoonkologischen Routine-Monitorings

R. Gastl ${ }^{1}$, D. Riedl ${ }^{1}$, E. Gamper ${ }^{2}$, C. Arnold ${ }^{1}$, D. Dejaco', G. Rumpold ${ }^{1}$

${ }^{1}$ Universitätsklinik für Medizinische Psychologie, Medizinische Universität Innsbruck, Innsbruck, Österreich

${ }^{2}$ Innsbruck Institute of Patient-reported Outcome Research (IIPCOR), Innsbruck, Österreich 
Einleitung: KrebspatientInnen berichten häufig aufgrund der Erkrankung und Behandlung unter körperlichen und psychosozialen Beeinträchtigungen zu leiden. Die Bewältigung solcher Belastungen im Zuge der ambulanten Strahlentherapie kann durch psychoonkologische Behandlung (PO) unterstützt werden.

Das Ziel der Studie war es zu untersuchen, wie viele PatientInnen zu Beginn der ambulanten Strahlentherapie einen Behandlungsbedarf aufweisen, bzw. den Wunsch nach PO äußern. Des Weiteren war das Ziel Behandlungspfade und Prädiktoren des PO abzubilden.

Methodik: Im Zuge einer retrospektiven Datenanalyse wurden die Ergebnisse eines routinemäßigen psychischen Belastungsscreenings zwischen 2015 und 2017 untersucht. Als Untersuchungsinstrument wurde das das Hornheide Screening Instrument (mit dem Cut-off $>4$ ) verwendet. Die Prädiktoren für den Behandlungswunsch wurden mittels schrittweiser binärer logistischer Regression identifiziert. In die Regression wurden soziodemographische und behandlungsbezogene Daten, aber auch die Items des Screening Instruments einbezogen.

Ergebnisse: Von den etwa 1700 ambulant bestrahlten onkologischen PatientInnen lagen von 944 PatientInnen ausgefüllte Screening-Fragebögen vor (Partizipationsrate ca. $55 \%$ ). Etwa $20 \%$ der inkludierten PatientInnen zeigten überdurchschnittliche Belastungswerte und $13 \%$ äußerten den Wunsch nach PO. Geringes emotionales Wohlbefinden $(\mathrm{OR}=11.3)$ und fehlende soziale Unterstuitzung $(\mathrm{OR}=9.4)$ waren starke Prädiktoren für den Behandlungswunsch. Ein substantieller Unterschied zwischen dem Grad der psychischen Belastung und dem Behandlungswunsch bei PatientInnen mit Pankreaskrebs, Hals-KopfTumoren sowie hämatoonkologischen Erkrankungen wurde beobachtet. Am häufigsten gaben PatientInnen mit Tumoren in den Harnorganen $(23.5 \%)$ und Lungenkrebs $(20.9 \%)$ einen psychoonkologischen Behandlungswunsch an.

Diskussion: Unsere Daten zeigen, dass direkt von den PatientInnen berichtete psychosoziale Probleme bessere Prädiktoren für den Wunsch nach PO-Behandlung waren als soziodemographische oder klinische Daten. Belastungsscreenings sollten daher in die klinische Routine implementiert werden. Die Verwendung von elektronischen Routinemonitoring könnte die Partizipationsrate verbessern.

\section{POS-06}

\section{Dreidimensionale (3D) multizelluläre Tumor- Normalgewebs-Sphäroide für die Strahlenbiologie - Vergleich der Strahlenreaktion von Sphäroiden mit normalen 2D Kultursystemen von Fibroblasten und Plattenepithelkarzinomen}

V. Kopatz ${ }^{1,2}$, G. Zemora ${ }^{1,2}$, S. Illedits ${ }^{1,3}$, W. Dörr ${ }^{1}$.

${ }^{1}$ Universitätsklinik für Strahlentherapie, Medizinische Universität Wien, Wien, Österreich

${ }^{2}$ Christian Doppler Labor für Medizinische Strahlenforschung für die Radioonkologie, Wien

${ }^{3} \mathrm{FH}$ Joanneum, Graz, Österreich

Einleitung: In den letzten Jahren haben sich 3D Zellkultursysteme, wie beispielsweise Sphäroide und in-vitro rekonstituierte Gewebe, immer mehr in der (Krebs-)Forschung durchgesetzt. Aufgrund ihrer dreidimensionalen Architektur dieser 3D Kultursysteme haben die Zellen ähnliche Wachstumsbedingungen wie in Geweben in vivo. Es entstehen ebenfalls Nährstoff-, Sauerstoff- und Metabolitgradienten sowie komplexe Zellinteraktionen und eine in-vivo ähnlichen Genexpression. Durch diese physiologischen Kulturbedingungen können 3D Modelle auch das natürliche Verhalten von Zellen auf Bestrahlung oder Behandlung mit therapeutischen Substanzen besser wiederspiegeln als Zellen in konventioneller 2D Monolayerkultur. Trotz dieses Vorteils den 3D Kulturen bieten, wird ein Großteil der heutigen (radiobiologischen) Forschung noch immer mit konventionellen 2D Zellkultursystemen durchgeführt.
Methodik: Für unsere weiteren radiobiologischen Studien haben wir daher ein 3D Sphäroidmodel aus normalen dermalen Fibroblasten (HSF1) und malignen Plattenepithelkarzinomzellen (FaDu) entwickelt und die Strahlenreaktion dieses 3D Models mit den Daten aus der 2D Kultur verglichen. Die zeit- und dosis-abhängige DNA Schädigung (bzw. Reparatur) wurde mittels Immunfluoereszenfärbung der yH2AX-Foci in 2D Kulturen bzw. in Paraffinschnitten von multizellulären Sphäroiden analysiert. Zusätzlich wurde für die FaDu-Zellinie ein Koloniebildungstest durchgeführt, um das klonogene Überleben der Zellen in 2D und 3D Kulturen nach Bestrahlung zu ermitteln.

Ergebnisse: HSF1 Zellen, die in 3D multizellulären Sphäroiden kultiviert wurden, zeigten eine ähnlich rasche DNA Reparatur wie unter 2D Bedingungen. Allerdings war die Fraktion der geschädigten Zellen sowie die Zahl der yH2AX-Foci/Nukleus wesentlich höher in 2DKulturen als in den Sphäroiden. Die klonogenen Überlebenskurven der FaDu-Zellen zeigten keinen signifikanten Unterschied in der Überlebensrate zwischen Zellen aus 2D und 3D Kulturen.

Diskussion: Erste Analysen zeigten gleichverlaufende Zellüberlebenskurven für FaDu-Zellen sowie ähnliche DNA-Schädigung/Reparatur in 2D und 3D Kulturen von HSF1 Zellen. Lediglich die Anzahl der geschädigten Zellen sowie die Anzahl der Foci pro Nucleus in den 2D Kulturen waren höher. Weitere vergleichende Studien zu genaueren Evaluierung der DNA-Schädigung/-Reparatur der FaDu Zellen sowie weitere Untersuchungen von radiobiologisch relevanten Faktoren, wie Hypoxie, Zellkontakten und differentialer Genexpression sind geplant.

\section{POS-07}

\section{Systemische Gabe von Heparin mildert radiogene Mukositis enoralis (Maus) durch die Interaktion mit den Entzündungs- und Repopulierungsprozessen ohne Stimulation vom Tumorwachstum}

\section{Kowaliuk, I. Schröder, V. Kopatz, P. Kuess, W. Dörr}

Universitätsklinik für Strahlentherapie, Medizinische Universität Wien, Wien, Österreich

Einleitung: Die vorliegende Studie untersucht den Effekt der systemischen Heparin-Behandlung auf die Ausprägung der radiogenen oralen Mukositis in einem etablierten Maus-Modell. Zusätzlich werden die möglichen zu Grunde liegenden Mechanismen untersucht. In einem 3D-Model wir auch getestet, ob die Heparin-Gabe einen stimulierenden Effekt auf den Tumor aufweist.

Methodik: Präklinische Untersuchungen erfolgten an C3H/Neu Mäuse. An Gruppen von je 50 Mäusen wurden unterschiedliche Bestrahlungsprotokolle in Kombination mit Heparin-Behandlung getestet. Die Substanzgabe erfolgte subkutan über variierende Zeitintervalle. Die Inzidenz und der zeitliche Verlauf der Schleimhautulzeration wurden als die klinisch relevanten Endpunkte analysiert.

Für mechanistische Studien wurde täglich fraktionierte Bestrahlung $(10 \times 3$ Gy) auf die Schnauzen der Mäuse appliziert. Heparin wurde täglich (Tag -3 bis 12) verabreicht. In zweitägigen Abständen wurden Gruppen von je 3 Mäusen eingeschläfert. Epitheliale Morphologie, Expression von Entzündungs-und Proliferationsmarker sowie von Zelladhäsionsmolekülen wurden untersucht.

Die Wachstum stimulierende Wirkung von Heparin wurde in 3DSphäroidkultur an FaDu-Zellen untersuch, alleine oder in Kombination mit Bestrahlung. Die Endpunkte waren dabei klonogenes Zellüberleben und Wachstum.

Ergebnisse: Die systemische Behandlung mit Heparin führte zu einer deutlichen Erhöhung der ED50-Werte, besonders in Kombination mit fraktionierter Bestrahlung. Des Weiteren wurden eine tendenzielle Verlängerung der Latenzzeit und eine deutliche Verkürzung der Ulzerationsdauer beobachtet. Mechanistische Studien ergaben, dass durch die Heparin-Gabe die Gesamtzahl der Epithelzellen sowie die Schicht- 
dicke im Laufe der zweiwöchigen Bestrahlung signifikant höher waren. Allerdings war die Proliferation der Epithelzellen durch Heparin nicht beeinflusst. Durch die Gabe von Heparin konnte die Expression der Entzündungsmarker NF- $\kappa \mathrm{B}$ und TNF $\alpha$ deutlich gesenkt werden; die Expression von Zelladhäsionsmolekül $\beta$ Catenin konnte gesteigert werden. Die in vitro Versuche mit FaDu-Sphäroiden ergaben, dass durch die Heparin-Gabe das Zellüberleben negativ beeinflusst und das Wachstum verlangsamt wurden.

Diskussion: Diese Studie liefert die ersten Hinweise auf einen mildernden Effekt von Heparin auf die Ausprägung der radiogenen oralen Mukositis. Der Mechanismus basiert, unter Anderem, auf der Linderung der Entzündungsreaktion und dem früheren Einsetzen der Repopulierung. Zudem führte die Heparin-Gabe zu keiner Wachstumsstimulation der Tumorzellen und erweist sich somit als eine klinisch relevante Behandlungsmöglichkeit. Weitere mechanistische Studien sowie in vitro Versuche mit weiteren Zelllinien und unterschiedlichen Behandlungsprotokollen sind geplant..

\section{POS-08}

\section{Three-dimensional organotypic squamous epithelium as models for the evaluation of the ionizing irradiation in normal and malignant tissues}

\section{G. Zemora ${ }^{1,2}$, W. Dörr ${ }^{1,2}$}

${ }^{1}$ Christian Doppler Laboratory for Medical Radiation Research for Radiation Oncology, Vienna, Austria

${ }^{2}$ Department of Radiotherapy,Medical University of Vienna, Vienna, Austria

Introduction: Radiotherapy, alone or in combination with surgery and/or chemotherapy is one of the most effective treatment options of cancer. Preclinical in vivo models are indispensable for radiobiological investigations. However, their application needs to follow the basic guidelines of animal studies (reduction, refinement, replacement) and such research should thus be supplemented by exploitation of suitable alternatives, e.g. in vitro systems. Three-dimensional (3D) organotypic culture systems have been shown to more accurately reflect the in vivo cell situation as compared to the standard two-dimensional (2D) monolayer cell culture. The development of 3D tissue models requires the ability to engineer tissues that mimic their in vivo counterparts. Yet, the majority of these 3D models are either lacking a proper microenvironment, a basement membrane or are just reconstituted exclusively from cancer cells, while the normal tissue cells within or surrounding the tumor formation are missing. We therefore generated human 3D squamous epithelial models that combine both normal and tumor cells in co-units, thus mimicking the situation in vivo. As such we have established a 3D complex oral squamous epithelium in vitro reconstruct and a 3D melanoma reconstruct.

Methods: The in vitro 3D squamous epithelium models consist of normal keratinocytes cultured over fibroblast-populated dermal matrices. In order to create co-cultures of both normal and malignant cells, 3D tumor spheroids were mixed with the normal keratinocytes. For the present study we used SCC25 squamous carcinoma spheroids and Lu1205 melanoma spheroids.

Results: Our results show positive IHC staining of the tumor cells that are surrounded by normal keratinocytes in a fully differentiated stratified epithelium.

Conclusion: These complex 3D organotypic systems can serve to test biologically targeted strategies to reduce normal tissue effects and/or to increase the tumor effects of ionizing radiation, before initiating preclinical in vivo or clinical studies.
POS-09

\section{Die intrafraktionelle Lagerungsgenauigkeit der Brainlab- Maske bei stereotaktischer intrakranieller Strahlentherapie}

J. Mangesius, R. Weigel, C. Arnold, D. Vasiljevic, I. Kvitsaridze, P. Lukas, U. Ganswindt, M. Nevinny-Stickel

Universitätsklinik für Strahlentherapie-Radioonkologie, Medizinische Universität Innsbruck, Innsbruck, Österreich

Einleitung: Wir untersuchten die intrafraktionelle Genauigkeit einer rahmenlosen thermoplastischen Maske zur Immobilisation des Kopfes bei der stereotaktischen Strahlentherapie von singulären intrakraniellen Tumoren. Bei der Anwendung nicht-invasiver thermoplastischer Masken ist von einer höheren intrafraktionellen Ungenauigkeit durch Bewegungen des Patienten auszugehen. Die in den meisten Studien verwendete prä- und postfraktionelle Messung ist nicht genau genug, um die intrafraktionelle Genauigkeit des Systems sicher bestimmen zu können.

Methodik: Die intrafraktionelle Ungenauigkeit wurde an 5 Patienten bei insgesamt 96 Fraktionen durch mehrfache ExacTrac-Messungen während der Bestrahlung, sowie vor und nach dieser, bestimmt. Es konnte somit die tatsächliche Positionsabweichung zum Zeitpunkt der Strahlenabgabe festgestellt werden. Die Patienten wurden mit der nicht-invasiven thermoplastischen Maske von BrainLAB immobilisiert. Es wurden im Median 6 Messungen pro Fraktion (Bereich 4 bis 11) erhoben. Durch Phantommessungen konnte der Einfluss gerätespezifischer Isozentrumsungenauigkeit minimiert werden. Die Daten wurden statistisch hinsichtlich der absoluten Abweichung in Translation und Rotation, sowie des Zusammenhangs zwischen vergangener Zeit und der gemessenen Ungenauigkeit analysiert.

Ergebnisse: Die zufälligen Fehler (SD) der Translationsabweichungen betrugen in der $\mathrm{x}, \mathrm{y}$ und $\mathrm{z}$ Achse jeweils $0,27 \mathrm{~mm}, 0,29 \mathrm{~mm}$ und 0,29 mm. Der 3D Vektor (3DV) hatte im Median eine Länge von 0,29 mm (Bereich 0-2,82 mm). 94,5\% der Messungen ergaben eine geringere 3D-Abweichung als $1 \mathrm{~mm}$. Es konnte eine Korrelation zwischen der vergangenen Zeit seit Beginn der Therapiesitzung und der $3 \mathrm{D}$ Abweichung gefunden werden $(\mathrm{rs}=0,45 p<0.01)$. Die 3D Vektorlänge stieg von $0,14 \mathrm{~mm}$ in den ersten zwei Minuten auf einen maximalen Wert von $0,53 \mathrm{~mm}$ nach 6 Minuten.

Diskussion: Prä- und postfraktionelle Messungen sind nicht ausreichend um die intrafraktionelle Abweichung genau abbilden zu können. Eine Verkürzung der Bestrahlungsdauer oder erneute Korrekturen während der Therapiesitzung könnten zu einer Verbesserung der Gesamtgenauigkeit führen. Die thermoplastische Maske bietet in Verbindung mit bildgestützter Navigation eine effektive interfraktionelle und intrafraktionelle Immobilisation für den Einsatz bei stereotaktischer intrakranieller Strahlentherapie.

\section{POS-10}

\section{Intestinal microbiota biomarkers associate with genotoxicity} but ameliorate radiation-induced bone loss

I. Maier ${ }^{1}$, J. Liu' ${ }^{1}$, PM. Ruegger ${ }^{2}$, J. Deutschmann ${ }^{3}$ JM. Patsch ${ }^{3}$, TH. Helbich ${ }^{3}$, J. Borneman ${ }^{2}$, RH. Schiestl ${ }^{1}$

${ }^{1}$ Department of Environm. Health Sciences, University of California, Los Angeles, Unites States

${ }^{2}$ Department of Plant Pathology and Microbiology, University of California, Riverside, California, Unites States

${ }^{3}$ Department of Biomedical Imaging and Image-guided Therapy, Medical University of Vienna, Vienna, Austria

Introduction: Restricted intestinal microbiota (RM) dysbiosis based on increased abundance of Lactobacillus johnsonii (LBJ) has previously been reported to promote elevated levels of systemic radiation-in- 
duced genotoxicity, proliferative lymphocyte activation, and polarization of metabolic pathways towards apoptosis.

Methods: Bacterial indicator phylotypes, including an unclassified Gram-negative bacteria, Barnesiella intestinihominis, and LBJ strains were found more abundant in wildtype RM mice and increased in prevalence after whole body irradiation in conventional microbiota (CM) mice.

Results: Muribaculum intestinale was found highest in female small intestines of RM mice, which were lacking Ureaplasma felinum. By contrast, Muribaculum intestinale was increased in male colons in the radiation-resistant CM phenotype. Endogenous LBJ strains influenced phylotype densities and reduced their abundance in irradiated $\mathrm{CM}$ mice compared to RM mice as assessed in intestinal mucosal cells, while the adaptive repair of chromosomal DNA lesions was induced more efficiently in $\mathrm{CM}$ than RM mice. Along with the reduced expression of tumor necrosis factor (TNF) in marrow, RM female mice showed improved micro-architectural bone structure compared with $\mathrm{CM}$ mice post particle radiation exposure.

Conclusion: Thus, genotoxic and anti-inflammatory traits in peripheral blood or tissue were associated with bacterial strains along with differential abundances of indicator phylotypes before and after radiation treatment.

\section{POS-11}

\section{Optische Kohärenztomographie des N. opticus als hilfreiche Untersuchung bei der lonentherapieplanung bei Patienten mit Tumoren des zentralen Nervensystems}

R. Dunavülgy ${ }^{2}$, C. Lütgendorf-Caucig ${ }^{1}$, U. Schmidt-Erfurth ${ }^{2}$, P. Georg ${ }^{1}$, D. Georg ${ }^{3}$, E. Hug $^{1}$

${ }^{1}$ MedAustron Ionentherapiezentrum, Wiener Neustadt, Österreich ${ }^{2}$ Universitätsklinik für Augenheilkunde und Optometrie,

Medizinische Universität Wien, Wien, Österreich

${ }^{3}$ Christian Doppler Labor für Medizinische Strahlenforschung für die Radioonkologie, Wien, Österreich

Einleitung: Die Untersuchung mittels optischer Kohärenztomographie (OCT) ist eine Standarduntersuchung in der modernen Ophthalmologie. Sie ermöglicht berührungs- und schmerzfrei die Untersuchung des hinteren Augenabschnitts (Macula und Sehnerv) innerhalb von wenigen Sekunden und wird hauptsächlich in der Diagnostik der altersbedingten Maculadegeneration sowie des Glaukoms eingesetzt. Es werden im Folgenden drei Patienten mit Tumoren des ZNS präsentiert, bei denen die Untersuchung des Sehnerven mittels OCT wichtige Informationen für die Bestrahlungsplanung liefert, welche mit den bis dato üblichen funktionellen Untersuchungen nicht zur Verfügung gestanden wären.

Methodik: Es werden im Folgenden die Krankengeschichten dreier Patienten präsentiert, welche sich im MedAustron (Wiener Neustadt) einer Protonentherapie aufgrund von Tumoren des ZNS unterzogen haben. Vor der Bestrahlungsplanung sowie im Follow-up wurde im Rahmen der ophthalmologischen Untersuchung bei jedem der drei Patienten eine Untersuchung des Sehnerven mittels OCT durchgeführt und dadurch zusätzliche Informationen gewonnen, welche aus planungstechnischer und forensischer Sicht sowie im Follow-up der Patienten nach Ionentherapie relevant waren.

Ergebnisse: Es wird die Krankengeschichte von drei Patienten mit Tumoren des ZNS (Meningeom) präsentiert (2 männlich, 1 weiblich). Alle drei Patientinnen und Patienten unterzogen sich vor der geplanten Ionenthearpie einer bzw. mehrerer Operationen. Bei jeweils einem männlichen und einem weiblichen Patienten gab die OCT-Untersuchung des Sehnerven wichtige Aufschlüsse über den Zustand der Sehnerven vor der geplanten Bestrahlung. Beim zweiten männlichen
Patienten ermöglichte die OCT-Untersuchung des Sehnerven das frühzeitige Entdecken einer Sehnervenschwellung und deren Therapie. Diskussion: Das OCT liefert schmerz- und berührungsfrei innerhalb kurzer Zeit wichtige Informationen über den Zustand des Sehnerven von Patienten mit Tumoren des ZNS. Diese Informationen können insbesondere bei der Bestrahlungsplanung als auch beim Follow-up dieser Patienten hilfreich sein.

\section{POS-12}

Effektivität von Single-Field Integrated Boost (SFIB) mit Spot Scanning Protonenbestrahlung bei Meningeomen

C. Lütgendorf-Caucig, G. Kragl, A. Perpar, J. Gora, P. Georg, B. Knäusl, U. Mock, R. Konstantinovic, C. Fussl, M. Stock, E. Hug

MedAustron Ionentherapiezentrum, Wiener Neustadt, Österreich

Einleitung: Simultan integrierte Boost-Verfahren über mehrere einzeloptimierte Felder bieten die Möglichkeit, die Dosis am Normalgewebe und den Behandlungszeitraum gegenüber sequenziellen Boost Techniken zu reduzieren.

Methodik: Die Zielvolumendefinition erfolgte auf Basis von CT, MR und DOTA-PET Untersuchungen. CTV1 beinhaltete das Meningeom (GTV per MR und PET), Hyperostosen und $5 \mathrm{~mm}-10 \mathrm{~mm}$ Margin entlang der Meningen. CTV2 (Boost) beinhaltete alleinig das Meningeom. Der PTV Margin betrug $3 \mathrm{~mm}$. Die Dosisvorschreibung für das PTV1 variierte je nach Risiko und WHO Grad zwischen 50,4 Gy(RBE) bis 54,0 Gy(RBE) und für das PTV2 50,4 Gy(RBE) bis 60,0 Gy(RBE) in $1,8 \mathrm{~Gy}-2 \mathrm{~Gy}(\mathrm{RBE})$ Einzeldosis. Die Protonentherapieplanung erfolgte für das Spot Scanning Verfahren basierend auf Single Field Optimierung (SFO) über mindestens 2 Einstrahlrichtungen, wobei ein Winkel von $>30^{\circ}$ zwischen den Feldern vorgegeben war. Die Planung erfolgte ICRU konform. Akute, therapiebedingte Nebenwirkungen wurden mittels CTCAE v.4.0 klassifiziert.

Ergebnisse: Zwischen 10/2017 und 08/2018 wurden insgesamt $30 \mathrm{~Pa}$ tienten mittels SFIB über 4 Felder (Range 2-6) geplant und behandelt. Das mediane Alter betrug 53,5 a (Range 27-81). Die Lokalisationen waren Schädelbasis $(80 \%, n=24)$, Falx $(10 \%, n=3)$, Tentorium $(6,7 \%, n=2)$ und Opticusscheide $(3,3 \%, n=1) ; 60 \%(n=18)$ WHO I, $16,7 \%(n=5)$ WHO II und 23,4 \% $(n=7)$ ohne Histologie. Das mediane PTV1 betrug 98,2 cm3 (Range 17,1 cm3-427,4 cm3), der mediane Boost (PTV2) 54,4 cm3 (Range 9,1 cm3-280,0 cm3). $80 \%(n=24)$ der Patienten hatten geringgradige akute Nebenwirkungen (Grad 1-2). Eine Grad 4 Nebenwirkung wurde beobachtet (einmaliges Anfallsgeschehen $74 \mathrm{~d}$ post RT).

Im Median wurden 27,5 Fraktionen (Range 27-30) pro Patient appliziert. Dies entspricht einer Verkürzung um 3 Tage (Range 2-6) gegenüber einem sequenziellen Boost. Die Zeit für die Bestrahlungsplanung als auch für die patientenspezifische Qualitätssicherung wurde durch die SFIB Planung gegenüber der sequenziellen Boost Planung deutlich reduziert.

Diskussion: Erste Behandlungsdaten zeigen, dass SFIB eine sichere und effiziente Technik in der Behandlung von Meningeomen darstellt. Diese Technik wird derzeit bereits bei Tumoren mit einem größeren Dosisdifferential zwischen CTV und GTV angewandt. Daraus resultiert eine größere Reduktion der Gesamtbehandlungszeit und die Möglichkeit der GTV Hypofraktionierung.

\section{POS-13}

\section{Ernährungsstatus bei Kopf-Hals Patienten unter Radiotherapie}

P. Clemens ${ }^{1}$, P. Szeverinski² ${ }^{2}$ C. Kreuter ${ }^{1}$, F. Böhler ${ }^{1}$, A. de Vries ${ }^{1}$

${ }^{1}$ Abteilung für Strahlentherapie und Radio-Onkologie,

Landeskrankenhaus Feldkirch, Feldkirch, Österreich 
${ }^{2}$ Institut für Medizinische Physik, Landeskrankenhaus Feldkirch, Feldkirch, Österreich

Einleitung: Die Ernährungssituation bei Patienten mit Kopf-Hals Plattenepithelkarzinomen ist bereits bei Diagnosestellung größtenteils unbefriedigend. Hinzu kommt durch die primär kurative Radio-Chemotherapie und deren Nebenwirkungen eine weitere Reduktion der Nahrungsaufnahme.

Wie verändert sich der Ernährungsstatus unter der Therapie und was für Parameter sollen zur Verlaufsbeurteilung herangezogen werden?

Methodik: Retrospektive Analyse von Patienten mit histologisch verifiziertem Plattenepithelkarzinom im Kopf-Hals Bereich, welche im Jahr 2017 an der Abteilung für Strahlentherapie und Radio-Onkologie im Landeskrankenhaus Feldkirch behandelt wurden. Insgesamt wurden 27 Patienten in die Analyse aufgenommen.

Die Parameter Gewicht $[\mathrm{kg}]$, Phasenwinkel $\left[^{\circ}\right]$, Fettmasse $[\mathrm{kg}]$ und Muskelmasse $[\mathrm{kg}]$ wurden mit einer Bioimpedanz Waage (Tanita MC780 MA, Tanita Europe) zu folgenden Zeitpunkten gemessen und in Korrelation zur Bestrahlung ausgewertet: Vor und am Ende der Bestrahlung (RTx) sowie 1 und 6 Monate nach der Bestrahlung. Die Auswertung erfolgte mittels SPSS (IBM).

Die applizierte Dosis war im Median 70,0 Gy (64,8 Gy bis 80,4 Gy), die Einzeldosis im Median betrug 1,9 Gy (1,2 Gy bis 2,3 Gy). Bei allen Patienten wurde VMAT angewendet. Bei Patienten mit Hyperfraktionierung $(n=2)$ wurde keine Chemotherapie appliziert, ansonsten wurde Cisplatin $(n=20)$, Mitomycin C/5-FU $(n=3)$ oder Cetuximab $(n=2)$ angewendet.

Ergebnisse: Die mediane Nachbeobachtungszeit war 136 Tage, 2 der 27 Patienten sind verstorben.

Folgende Mittelwerte $( \pm$ SD) zeigten jeweils einen signifikanten Unterschied $(p<0,05)$ zu den definierten Zeitpunkten (Tab. 1).

Diskussion: Zusammenfassend zeigt unsere Auswertung eindeutig, dass sich die Ernährungssituation unter der Radiotherapie verschlechtert. Zur Beurteilung des Ernährungsstatus im klinischen Alltag können alle untersuchten Parameter herangezogen werden. Es kann bis einen Monat nach der Radiotherapie ein Verlust in allen Parametern nachgewiesen werden. Erst bei den 6 Monatskontrollen zeigen sich alle Werte bis auf die Fettmasse wieder verbessert.

Vor allem die Frage ob einer Verschlechterung der Ernährungssituation entgegen gewirkt werden kann ist offen uns sollte dringend prospektiv untersucht werden.

\section{POS-14}

\section{Kombinierte adaptive Photonen-Protonen Therapie bei Kopf- Hals Tumoren}

H. Herrmann ${ }^{1}$, A. Perpar ${ }^{2}$, M. Schmid ${ }^{1}$, P. Georg ${ }^{2}$, M. Stock ${ }^{2}$, G. Kragl ${ }^{2}$, G. Altorjai ${ }^{1}$, F. Baier ${ }^{1}$, J. Widder ${ }^{1}$, E. Hug $^{2}$

${ }^{1}$ Universitätsklinik für Strahlentherapie, Medizinische Universität Wien, Wien, Österreich

${ }^{2}$ MedAustron Ionentherapiezentrum, Wiener Neustadt, Österreich

Einleitung: PatientInnen mit Nasopharynxkarzinom bzw. sinu-nasalem Karzinom, insbesondere bei intrazerebraler Beteiligung, stellen auf Grund der Nähe zu kritischen Strukturen wie dem optischen System eine große Herausforderung in der Radiotherapie dar. Eine rezente
Metaanalyse zeigte, dass die Anwendung der Partikeltherapie hierbei $\mathrm{zu}$ verbesserten Therapieergebnissen gegenüber einer alleinigen Photonentherapie führt.

Methodik: Als Zusammenarbeit zwischen MedAustron und MedUni Wien wurde ein Protokoll zur kombinierten Photonen-/Protonentherapie bei fortgeschrittenen Kopf-Hals Tumoren erstellt. Einschlusskriterium ist das Vorliegen eines Schädelbasis-nahen Karzinoms bei gleichzeitiger Notwendigkeit einer Bestrahlung der Halslymphknoten +/- konkomitanter Chemotherapie. Eine medizinische Indikation zu kombinierter Photonen und Protonen Behandlung besteht dann, wenn neben Empfehlung des Kopf-Hals Tumorboards mindestens einer der folgenden Punkte zutrifft:

1) Die Einhaltung der geforderten Dose-volume-constraints kritischer Risikostrukturen ist bei einer Zieldosis von 70-74 Gy mit einer alleinigen Photonentherapie (mittels IMRT oder VMAT Technik) nicht möglich

2) Kritische Risikostrukturen werden mit alleiniger Photonentherapie deutlich höher belastet.

3) Die kombinierte Photonen-Protonentherapie führt zu einer signifikant verbesserten Dosisabdeckung des Zielvolumens.

Die Indikation zur kombinierten Photonen-Protonentherapie wird mittels Planvergleich verifiziert.

Ergebnisse: Die Behandlung wird in 3 Phasen durchgeführt, wobei ein adaptives Therapiekonzept verfolgt und der Bestrahlungsplan jeweils anhand repetitiver Bildgebung optimiert wird.

- Phase I beinhaltet die Behandlung an MedUni Wien-AKH (Tumor und Halslymphknoten): Photonentherapie im Bereich des Primärtumors und der regionären Lymphknotenstationen bis 54 Gy bei 1.8 Gy ED mit gegebenenfalls SIB im Bereich von befallenen Lymphknoten +/- konkomitanter systemischer Therapie

- Phase II und III: Protonenbehandlung im Bereich des Primärtumors am MedAustron bis kumulativ 66 Gy im Bereich der initialen Tumorausdehnung (Phase II) und bis 74 Gy im Bereich des Resttumors nach Photonentherapie (Phase III)

Ergebnisse der ersten PatientInnen werden gezeigt werden.

Diskussion: Durch die Anwendung einer kombinierten PhotonenProtonentherapie wird eine Dosiseskalation für diese herausfordernden Tumoren ermöglicht. Dadurch soll die Wahrscheinlichkeit einer langfristigen lokalen Kontrolle bei gleichzeitig reduziertem Nebenwirkungsprofil gesteigert werden.

\section{POS-15}

\section{Survival in early lung cancer patients treated with DART or SBRT depends on co-morbidity}

F. Zehentmayr ${ }^{1}$, M. Sprenger ${ }^{2}$, L. Rettenbacher ${ }^{3}$, R. Wass ${ }^{4}$, P. Porsch ${ }^{4}$, G. Fastner ${ }^{1}$, C. Pirich ${ }^{3}$, M. Studnicka ${ }^{4}$, F. Sedlmayer ${ }^{1}$

${ }^{1}$ Universitästklinik für Radio-Onkologie und Radiotherapie, Uniklinikum Salzburg-Landeskrankenhaus, Salzburg, Austria ${ }^{2}$ Institut füpr Sozialmedizin und Epidemiologie, LKH-Univ.Klinikum Graz, Graz, Austria

${ }^{3}$ Universitätsklinik für Nuklearmedizin und Endokrinologie, Uniklinikum Salzburg-Landeskrankenhaus, Salzburg, Austria ${ }^{4}$ Universitätsklinik für Pneumologie, Uniklinikum SalzburgLandeskrankenhaus, Salzburg, Austria

Tab. 1

\begin{tabular}{lllll}
\hline & (RTx Beginn & vs. RTx Ende & vs. 1 M. nach RTx & vs. 6 M. nach RTx) \\
\hline Gewicht: & $(75,9 \pm 14,2) \mathrm{kg}$ & vs. $(73,0 \pm 11,1) \mathrm{kg}$ & vs. $(69,9 \pm 10,1) \mathrm{kg}$ & vs. $(70,1 \pm 10,3) \mathrm{kg}$ \\
Phasenwinkel: & $(4,6 \pm 1,0)^{\circ}$ & vs. $(4,3 \pm 1,0)^{\circ}$ & vs. $(4,1 \pm 0,9)^{\circ}$ & vs. $(4,3 \pm 1,1)^{\circ}$ \\
Fettmasse: & $(17,1 \pm 9,2) \mathrm{kg}$ & vs. $(15,6 \pm 6,9) \mathrm{kg}$ & vs. $(14,8 \pm 7,2) \mathrm{kg}$ & vs. $(12,8 \pm 4,7) \mathrm{kg}$ \\
Muskelmasse: & $(55,8 \pm 8,7) \mathrm{kg}$ & vs. $(54,7 \pm 6,9) \mathrm{kg}$ & vs. $(51,8 \pm 6,0) \mathrm{kg}$ & vs. $(52,9 \pm 6,9) \mathrm{kg}$ \\
\hline
\end{tabular}


Introduction: $10 \%$ of the lung cancer patients are diagnosed in stages I-IIa. Pathological proof of disease cannot always be obtained due to comorbidity or reluctance to undergo invasive diagnostic procedures. In the current study survival data of patients with and without pathological proof are compared. We hypothesized that improved survival in the latter group could mask overtreatment of these patients.

Methods: 163 patients with NSCLC I-IIa treated between 2002 and 2016 were eligible. 123/163 (75\%) had pathological confirmation of disease, whereas 40/163 (25\%) patients had not. In accordance with international guidelines, both groups received high dose radiotherapy, either as dose differentiated accelerated radiotherapy (DART) or stereotactic body radiotherapy (SBRT). Co-morbidity was assessed with the Charlson score (CS).

Results: Median follow-up was 25,2 months (range 0,3-162). 96/163 (59\%) patients died: 48/163 (29\%) cancer related deaths occurred and 48/163 (29\%) patients died for other reasons. 67/163 (41\%) patients are still alive. Median overall survival (OS) for patients without pathological confirmation was 39,4 months (range 0,5-162), which did not differ from those with microscopical proof of disease (40,1 months; range $0,3-147,5$; log-rank $p$-value $=0,958)$. The median cancer specific survival (CSS) of 113,4 months (range 0,5-162) in the non-confirmation group did not differ either (log-rank $p$-value: 0,585 ) when compared to 51,7 months (range 3,7-129,5) in patients with pathology. In the Cox regression, a CS of 3 or higher was the only significant prognosticator prognostic factor $(p=0,026 ; \mathrm{HR}=2,1$ range $1,1-4,1)$.

Conclusion: OS and CSS in early lung cancer patients depend on comorbidity rather than on pathological confirmation of disease.

\section{POS-16}

\section{Pulmonary function remains constant after DART in patients with NSCLC stage III}

F. Zehentmayr ${ }^{1}$, F. Wolf ${ }^{1}$, J. Kaiser ${ }^{1}$, S. Windischbauer ${ }^{1}$, P. Kopp ${ }^{1}$, G. Fastner ${ }^{1}$, R. Wass ${ }^{2}$, P. Porsch ${ }^{2}$, M. Studnicka, F. Sedlmayer ${ }^{1}$

${ }^{1}$ Universitästklinik für Radio-Onkologie und Radiotherapie, Uniklinikum Salzburg-Landeskrankenhaus, Salzburg, Austria ${ }^{2}$ Universitätsklinik für Pneumologie, Uniklinikum SalzburgLandeskrankenhaus, Salzburg, Austria

Introduction: Radiation dose escalation strategies for NSCLC stage III patients are said to be associated with pulmonary toxicities that are potentially higher than with concomittant chemoradiotherapy, which is regarded as standard of care [1]. The aim of the current study was to investigate pulmonary function changes within six months after accelerated high-dose radiotherapy.

Methods: 44 patients with NSCLC stage III treated between January 2015 and December 2017 were eligible for the current analysis. After two cycles of platinum-based induction chemotherapy, all patients received dose differentiated accelerated radiotherapy (DART) with two daily fractions of 1,8 Gy each and total doses of 73,8 Gy to 90,0 Gy. Pulmonary function tests (PFT) were performed before (=baseline) and after radiation treatment. These tests were continued on a 3-monthly basis at each follow-up visit. FEV1 and DLCO as representative markers for lung function impairment (either restrictive or obstructive) were analyzed.

Results: With a minimum follow-up of 6 months, a median OS of 24,0 months (range: 12,6-35,4 months) was achieved. For $42 / 44$ (95\%) patients the ratio between the PFTs at the 6-months follow-up visit compared to baseline was close to 1: median FEV1-ratio 1,0 (range: 0,61,9), median DLCO-ratio 1,0 (range: 0,8-2,2).

Conclusion: Patients who receive sequential accelerated chemoradiotherapy (DART) for stage III NSCLC retain pre-treatment lung function.
POS-17

\section{Eine retrospektive Studie: Vergleich von 3D-CRT und VMAT in Bezug auf die kraniospinale Bestrahlung}

\author{
A. Pölzl ${ }^{1}$, P. Steffens ${ }^{1}$, C. Zisser ${ }^{2}$, U. Temmel ${ }^{2}$, B. Zurl ${ }^{2}$.
}

${ }^{1}$ FH Joanneum Graz, Radiologietechnologie, Graz, Österreich ${ }^{2}$ Univ. Klin. f. Strahlentherapie-Radioonkologie, LKH-Univ.Klinikum Graz, Graz, Österreich

Einleitung: Die kraniospinale Bestrahlung stellt einen wesentlichen Bestandteil des Therapiekonzeptes zur Behandlung von ZNS-Tumoren dar. Dabei ist in der klinischen Routine derzeit die 3D-konformale Radiotherapie (3D-CRT) als Bestrahlungstechnik etabliert. Durch die Entwicklung neuer Verfahren und Techniken stellt die volumetrisch modulierte Arc Therapie (VMAT) eine weitere Bestrahlungsmöglichkeit dar. Methodik: Es wurde eine retrospektive Datenanalyse von Bestrahlungsplänen der 3D-CRT und VMAT im Hinblick auf die Dosiskonformität und -homogenität im Zielvolumen sowie die Dosisbelastung im umliegenden Gewebe bei der kraniospinalen Bestrahlung durchgeführt. Dazu wurden für die 3D-CRT und VMAT jeweils fünf Bestrahlungspläne retrospektiv herangezogen, in welchen anschließend die Konturierung interessierender Volumina vorgenommen wurde. In weiterer Folge wurden Auswertungen und eine Analyse der Daten durchgeführt.

Ergebnisse: Für die VMAT wurde ein durchschnittlicher Konformitätsindex $(\mathrm{CI})$ von 1,1 ermittelt, wobei dieser Wert unter Verwendung der 3D-CRT bei 1,8 lag. Der durchschnittliche Homogenitätsindex (HI) betrug bei der VMAT 0,09 und bei der 3D-CRT 0,19. Im Hinblick auf die Dosisbelastung im umliegenden Gewebe konnte durch die VMAT eine Reduktion der durchschnittlichen Dmean bei 57 \% der Risikoorgane erreicht werden. Außerdem wurde unter Verwendung der VMAT eine Abnahme der durchschnittlichen Dmax in allen Risikoorganen, mit Ausnahme des Larynx, verzeichnet. Des Weiteren wurden beim Körperstamm im Vergleich zwischen 3D-CRT und VMAT prozentuelle Anteile von $3 \%$ vs. $0 \%$ für das V20Gy, $9 \%$ vs. $7 \%$ für das V10Gy, $14 \%$ vs. $36 \%$ für das V5Gy und $21 \%$ vs. $86 \%$ für das V2Gy ermittelt. Das V20Gy im Bereich von Körperstamm und Schädel lag bei der 3D-CRT bei $19 \%$ und bei der VMAT bei $7 \%$.

Diskussion: Durch die kraniospinale Bestrahlung mittels VMAT kann eine bessere Dosiskonformität und -homogenität im Zielvolumen im Vergleich zur 3D-CRT erzielt werden. Des Weiteren ergab sich unter Verwendung der VMAT eine größere Schonung von umliegenden Gewebe im Hinblick auf die durchschnittliche mittlere und maximale Dosis, wobei auch der Hochdosisbereich deutlich reduziert werden konnte. Jedoch weist die VMAT-Technik auch einen hohen Niedrigdosisbereich auf. Trotz dessen, kann durch die Betrachtung der Ergebnisse ein Vorteil für die Bestrahlung mittels VMAT abgeleitet werden.

\section{POS-18}

\section{Untersuchung von 3 Behandlungstechniken bei adjuvanter Bestrahlung der Brust unter Einschluss der Supraklavikulärregion}

M. Preihs, B. Zurl, C. Zisser, C. Trifterer, U. Temmel, C. Kaltenegger, D. Kadija, K. Kapp

Univ. Klin. f. Strahlentherapie-Radioonkologie, LKH-Univ.Klinikum Graz, Graz, Österreich

Einleitung: Brustbestrahlungen stellen im Zeitalter von intensitätsmodulierten Rotationstechniken nach wie vor eine Herausforderung dar, da zusätzlich zur Schonung der eng benachbarten Risikoorgane besondere Rücksicht auf die kontralaterale Brust genommen werden muss. Um hier strahleninduzierte Sekundärmalignome zu vermeiden werden nach wie vor tangentiale Techniken bevorzugt. 
Methodik: Für 7 Patientinnen mit linksseitigem Brustkrebs wurden 3 verschiedene Bestrahlungstechniken im Bestrahlungsplanungssystem Pinnacle (Philips Medical Systems) gerechnet. Alle 3 Techniken verwendeten tangentiale Felder für die Brustbestrahlung entweder 3D konformal oder als hybrid IMRT. Für den supraklavikulären Anteil wurden entweder bei gleichem Isozentrum konformale Photonenfelder (3D-konformal) oder intensitätsmodulierte Photonenfelder (hIMRT-Technik) oder eine Kombination aus Photonen und Elektronenfeldern (Ph-El Technik) verwendet.

Zum Vergleich wurden die Dosis-Volumen-Histogramme (DVH) von Herz, Lunge und kontralateraler Brust erfasst, der Konformitätsindex (CI) des Planungszielvolumens (PTV) mit CI=VPTV95\%/VPTV sowie der Homogenitätsindex im PTV (HI) mit HI=(D2 \%-D98 \%)/D50 \%.

Ergebnisse: Für die 3 Techniken 3D-konformal, hIMRT und Ph-El wurden im Mittel 519 (474-613), 559 (542-585) und 459 (418-569) Monitoreinheiten errechnet. Bei der hIMRT zeigte der mittlere Konformitätsindex von 1.15 (1.044-1.204) den niedrigsten Wert im Vergleich zu Ph-El von 1.222 (1.101-1.281) und zur 3D-konformalen Technik von 1.29 (1.137-1.436). Auch der mittlere Homogenitätsindex war für hIMRT mit 0.116 und für die 3D-konformale Technik mit 0.14 gering, während die PH-El Technik einen doppelt so hohen Wert aufwies. Die mittlere Herzdosis von 4.4 Gy $(3.0-6.3)$ und die mittlere ipsilaterale Lungendosis von 14.5 Gy (9.9-18.2) waren für alle Techniken gleich. Die geringste mittlere Dosis in der kontralateralen Brust von 0.491 Gy wurde bei der $\mathrm{Ph}-\mathrm{El}$ Technik erzielt.

Diskussion: Der Vergleich von 3 tangentialen Techniken zur Bestrahlung der Brust und der Supraklavikulärregion zeigte eine deutlich höhere Konformität und Homogenität bei intensitätsmodulierten Photonenfeldern. Die Dosis in den Risikoorganen Herz, Lunge und kontralateraler Brust war von der verwendeten Technik unabhängig.

\section{POS-19}

\section{MV oder kV Aufnahmen für die bildgeführte Einstellung der clipmarkierten Mamma-Tumorbettbestrahlung?}

\section{F. Beck, P. Wagner, J. Fuchs, N. Zagler, M. Metz}

Institut für Radioonkologie und Strahlentherapie, Landesklinikum Wiener Neustadt, Wiener Neustadt, Österreich

Einleitung: Die Verifikation der korrekten Patienten-Lagerung spielt eine sehr wichtige Rolle in der Strahlentherapie. Die Art der Verifikationsmethode ( $\mathrm{MV}, \mathrm{kV}, \mathrm{CBCT}$, etc.) hängt davon ab, welche anatomischen Strukturen zur Beurteilung der korrekten Lagerung herangezogen werden.

Die jahrelang etablierte Verifikationsmethode bei der Tumorbettbestrahlung des Mammakarzinoms mit Photonen war die MV Bildgebung. Da in der MV-Aufnahme die Tumorbettclips nicht sichtbar sind, haben wir zusätzlich eine $\mathrm{kV}$-Bildgebung verwendet.

Methodik: Bei 57 Patientinnen wurden bei jeder Bestrahlung des Tumorbettes MV und kV-Aufnahmen zur Verifikation der Lagerung durchgeführt. Die beiden Modalitäten wurden miteinander verglichen. Mittels MV-Bildgebung können Lunge, Bodykontur und Mamille beurteilt werden. Für die Beurteilung des clipmarkierten Tumorbettes muss die $\mathrm{kV}$-Bildgebung verwendet werden.

Im Rahmen der Auswertung wurden die Unterschiede der beiden Bildgebungsmodalitäten hinsichtlich Durchführung, Bildqualität sowie etwaige Probleme samt Lösungen, behandelt.

Ergebnisse: Es zeigt sich in $11 \%$ der Fälle eine maximale Abweichung von $0.3 \mathrm{~cm}$. In $23 \%$ liegt die Divergenz bei min. $0.4 \mathrm{~cm}$ und max. $1.6 \mathrm{~cm}$. Diese größeren Abweichungen begründen sich durch die Veränderung des Brustgewebes während der vorangegangenen Bestrahlungssitzungen, atembedingt, bewegungsbedingt, mangelhafte Qualität der Verifikationsaufnahmen und nicht eindeutige Erfassung der Clips. In den restlichen $67 \%$ der Fälle stimmten die für die Verifikation der Lagerung relevanten Strukturen überein.

Diskussion: Für die Darstellung der Tumorbettclips muss die moderne $\mathrm{kV}$-Bildgebung verwendet werden!

\section{POS-20}

\section{D-gedruckte Moulagen in der Brachytherapie}

\section{J. Soltner ${ }^{1}$, H. Ritschl ${ }^{2}$, A. Nischelwitzer ${ }^{3}$, A. Fallast ${ }^{4}$, B. Zurl ${ }^{1}$}

${ }^{1}$ Univ. Klin. f. Strahlentherapie-Radioonkologie, LKH-Univ.Klinikum Graz, Graz, Österreich

${ }^{2}$ FH Joanneum Graz, Radiologietechnologie, Graz, Österreich ${ }^{3} \mathrm{FH}$ Joanneum Graz, Informationsmanagement, Graz, Österreich ${ }^{4} \mathrm{FH}$ Joanneum Graz, Luftfahrt/Aviation, Graz, Österreich

Einleitung: Der 3D-Druck etabliert sich immer mehr in der Medizin. Anwendungen in der Strahlentherapie wurden bereits in Studien über die Anfertigung verschiedenster Hilfsmittel in der Teletherapie oder Kontakttherapie beschrieben. In der Brachytherapie besteht somit die Möglichkeit, die Lage der Strahlungsquelle in gutsitzenden oberflächlichen Moulagen vorzuplanen. Es wurde die 3D-Datei für eine Moulage entwickelt und verschiedene Druckmaterialien hinsichtlich des Patientenkomforts und der Innenraumbefüllung mit Hilfe kommerzieller 3D-Drucker untersucht.

Methodik: Der CT-Datensatz eines Phantoms diente als Basis für die Konstruktion der Moulage. Nachdem die Moulage im Bestrahlungsplanungssystem konturiert wurde, mussten die Applikatoröffnungen zusätzlich definiert werden. Für die Untersuchung der Druckmaterialien wurden Testobjekte aus ABS (Reprap Austria) und flexiblem Kunstharz (Formlabs) mit unterschiedlicher Dichte 3D-gedruckt. Die Dichte der Moulage wird bei FDM-Druckern direkt in der Drucksoftware definiert. Dabei kann das Design des Innenraums automatisch oder manuell bestimmt werden. Bei Druckern der SLA-Technologie muss das Innere manuell konstruiert werden. CT-Datensätze des Phantoms mit den unterschiedlich dichten Moulagen dienten als Grundlage der Brachytherapie-Bestrahlungsplanung, die mit Thermolumineszenzdosimetern überprüft wurde.

Ergebnisse: Der Entwicklungsprozess der 3D-Datei besteht aus der virtuellen Konstruktion der Moulage auf einem CT-Datensatz in der Bestrahlungsplanungssoftware (Pinnacle3 v.16, Philips), der anschlieBenden Nachbearbeitung und der Speicherung als STL-File. Dabei kann die Quellenposition anwenderspezifisch über die Position der Applikatoröffnungen bestimmt werden. Die unterschiedlichen Dichten und Innenraumbefüllungen wurden dosimetrisch geprüft. Bei Betrachtung der Materialien wiesen gummiartige Strukturen, wie flexibles Kunstharz, eine glattere Oberfläche auf und versprechen somit einen höheren Patientenkomfort.

Diskussion: Patientenspezifische Moulagen können in Zukunft mit Hilfe eines 3D-Druckers erstellt werden, wobei durch die zuvor geplanten Applikatoröffnungen eine erleichterte reproduzierbare Planung ermöglicht wird. Bei der Moulagenerstellung ist auf das verwendete Material und auf die Objektdichte hinsichtlich des Patientenkomforts und der Bestrahlungsplanung zu achten.

\section{POS-21}

\section{Aktuelle zahnärztliche Sanierungskonzepte vor Strahlentherapie im Kopf-Hals-Bereich}

\section{S. Schneider ${ }^{1}$, G. Altorjai ${ }^{2}$}

${ }^{1}$ Universitätsklinik für Mund-, Kiefer- und Gesichtschirurgie, Medizinische Universität Wien, Wien, Österreich

${ }^{2}$ Universitätsklinik für Strahlentherapie, Medizinische Universität Wien, Wien, Österreich 
Einleitung: Trotz moderner Therapieverfahren kommt es im Rahmen einer Strahlentherapie immer zur Schädigung gesunder Gewebestrukturen und Funktionsstörungen umliegender Organe. Die daraus resultierenden akuten, teils passageren Nebenwirkungen betreffen aus zahnärztlicher Sicht insbesondere die Mundschleimhäute und Speicheldrüsen; Strahlenkaries und (infizierte) Osteoradionekrose (ORN) stellen hingegen chronische Folgen einer Radiotherapie dar.

Methodik: Durch eine möglichst frühzeitige Diagnostik und Therapieplanung unter individueller Risikoabwägung können derartige Komplikationen häufig reduziert werden. Das etablierte präradiotherapeutische Betreuungskonzept besteht dabei in der chirurgischen, parodontologischen und konservierenden Vorbehandlung, ausführlichen Mundhygieneinstruktionen und der fallweisen Anfertigung von Strahlenschutz- bzw. Fluoridierungsschienen. Besonderen Stellenwert nimmt die Fokussuche ein: Potenzielle Herde müssen identifiziert und vor Beginn der Strahlentherapie beseitigt werden. Zudem sollten bereits vorhandene prothetische Versorgungen hinsichtlich ihrer Passung überprüft und ggf. optimiert werden. Zudem ist bereits in dieser Phase eine später notwendige prothetische (Neu-) Versorgung im Sinne eines Backward-Plannings zu berücksichtigen.

Ergebnisse:Die intensitätsmodulierten Radiotherapie (IMRT) ermöglicht die exakte Anpassung der Dosisverteilung an das Tumorgebiet mit dem Vorteil einer geringeren Strahlenbelastung bei zugleich größtmöglicher Schonung des stomatognathen Systems. Dies führt neben einer deutlichen Reduktion der strahlenbedingten Nebenwirkungen auch zu einem Rückgang der Folgekomplikationen. Unter diesen Aspekten wurden die bisherigen präradiotherapeutischen Sanierungskonzepte im Sinne einer zurückhaltenderen, risikoadaptierten Zahnsanierung (RaZS) angepasst. Die bestehenden Richtlinien wurden dabei grundsätzlich beibehalten: Vordefinierte IMRT-Hochdosisgebiete werden weiterhin herkömmlich konservierend-chirurgisch saniert. In Bereichen mit einer geringeren Dosis und einem dementsprechend niedrigen oder mittleren Risiko kann jedoch bei der Sanierung zurückhaltender verfahren werden.

Diskussion: Dieses Vorgehen hat zahlreiche Vorteile: der damit verbundene Erhalt der Lebensqualität des Patienten stellt ein wichtiges Ziel der modernen Strahlentherapie dar. Zudem ist die später häufig notwendige prothetische Rehabilitation unter Einbeziehung der verbliebenen Restbezahnung deutlich einfacher umsetzbar.

\section{POS-22}

Bestrahlungsplanung mittels zusätzlichem PET/CT im Vergleich zur alleinigen CT-/MR-Bildgebung bei primären Hirntumoren. Ergibt sich daraus ein klinischer Mehrwert?

\section{N. Jawadi ${ }^{1}$, P. Steffens ${ }^{2}$, K. S. Kapp ${ }^{1}$, R. Part ${ }^{1}$}

${ }^{1}$ Univ. Klin. f. Strahlentherapie-Radioonkologie, LKH-Univ.Klinikum Graz, Graz, Österreich

${ }^{2}$ FH Joanneum Graz, Radiologietechnologie, Graz, Österreich

Einleitung: Die Magnetresonanztomographie (MRT) ist die etablierte Methode zur Definition des Bestrahlungszielvolumens. Gemeinsam mit einer Computertomographie (CT) bildet sie die Grundlage für die Bestrahlungsplanung und Dosisberechnung. Die Positronen-Emissions-Tomografie (PET) wird bei Hirntumoren ebenfalls eingesetzt. Denn während die CT nur eine örtliche Lokalisation der Organe und des Tumors ermöglicht, können mittels PET Stoffwechselfunktionen dargestellt werden.

Methodik: In dieser theoretischen Literaturarbeit (Bachelorarbeit) sollte untersucht werden, inwiefern die Hybridbildgebung mittels PET-CT einen klinischen Mehrwert in der Zielvolumenkonturierung von primären Hirntumoren verglichen mit einer alleinigen CT/MRT- Bildgebung hat. Die Literaturrecherche wurde auf online Datenbanken wie ResearchGate, Google Scholar, Medline und Pubmed durchgeführt. Als Suchbegriffe wurden „PET/CT role in radiotherapy planning“ und „PET/CT in radiotherapy treatment planning of brain tumors" verwendet.
Ergebnisse: Die Integration von PET/CT in die Zielvolumenkonturierung führte bei PatientInnen mit Gliomen zu einer Vergrößerung des Tumorvolumens (=GTV). Die Differenz zwischen dem mittleren GTV (MRT/CT) und dem GTV (PET/CT) lag in den Studien zwischen 3.7-7.5 ccm. Der Einfluss der PET/CT auf das GTV war bei PatientInnen mit High-Grade Gliomen signifikant $(p=0.04)$, bei Low-Grade Gliomen konnte keine eindeutige Aussage getroffen werden. Für PatientInnen mit Meningeomen war das GTV (MRT/CT) bei $75 \%$ der ProbandInnen größer als GTV (PET/CT). Darüber hinaus konnten die Daten durch die Integration einer PET/CT in die Bestrahlungsplanung die Interobserver-Variabilität reduzieren und die mittlere Überlebenszeit von 6 auf 11 Monate erhöhen.

Diskussion: Die Integration der PET/CT in die Bestrahlungsplanung von High-Grade Gliomen erscheint sinnvoll. Um den Einfluss des PET/CT bei Low-Grade Gliome und Meningeomen besser analysieren zu können, werden Studien mit größeren Fallzahlen benötigt. Weiters sollte die PET/CT aufgrund der Reduktion der Interobserver-Variabilität und der Gewährleistung der Reproduzierbarkeit der Zielvolumendefinition in die Bestrahlungsplanung integriert werden.

\section{POS-23}

\section{Vergleich einer präoperativen versus postoperativen MRT-basierten PTV-Konturierung: In silico Auswertung bei PatientInnen mit Glioblastomen}

\author{
N. Jawadi ${ }^{1}$, P. Steffens ${ }^{2}$, K. S. Kapp ${ }^{1}$, R. Partl ${ }^{1}$
}

${ }^{1}$ Univ. Klin. f. Strahlentherapie-Radioonkologie, LKH-Univ.Klinikum Graz, Graz, Österreich

${ }^{2}$ FH Joanneum Graz, Radiologietechnologie, Graz, Österreich

Einleitung: Das Glioblastom zählt zu den häufigsten und aggressivsten primären Hirntumoren mit einer generell schlechten Prognose. Eine postoperative Radiotherapie +/- konkomitanter Chemotherapie verbessert das Outcome. Die Frage, ob als Basis für das Planungszielvolumen (PTV) eine prä- oder postoperative Bildgebung herangezogen werden sollte und ob jeweils nur der klinisch erkennbare Tumor bzw. das Tumorbett oder zusätzlich das perifokale Ödems einbezogen werden sollte, wird von den Fachgesellschaften kontroversiell gesehen. Je nach gewähltem Konzept hat dies jedoch großen Einfluss auf das Behandlungsvolumen.

Methodik: Im Rahmen einer Pilotstudie wurde bei 10 PatientInnen mit Glioblastomen ein präoperatives Konzept und ein postoperatives Konzept (RTOG 0825) in silico miteinander verglichen und hinsichtlich PTV-Veränderungen ausgewertet.

Ergebnisse: Der Vergleich zwischen den beiden Konzepten zeigte in Bezug auf das PTV1 keine signifikanten Unterschiede (CI $95 \%$ : $-59,42 \mathrm{ccm}-132,99 \mathrm{ccm}, p=0,41)$. Jedoch in Bezug auf das PTV2 ergab sich folgende Änderung: Das durchschnittliche Volumen des präoperativen PTV2 betrug $111,41 \mathrm{ccm} \pm 69,66 \mathrm{ccm}$ (SD). Für das postoperative PTV2 wurde ein Mittelwert von $282,10 \mathrm{ccm} \pm 92,71 \mathrm{ccm}$ (SD) berechnet. Das postoperative RTOG-Konzept führte folglich zu einer signifikanten Vergrößerung des PTV2 um 170,68 cm3 (CI95\%: $86,23 \mathrm{ccm}-255,14 \mathrm{ccm}, p=0,001)$.

Diskussion: Die operationsbedingten Veränderungen führten zu erheblichen Volumenunterschieden. Mit dem postoperativen Konturierungskonzept konnte für das PTV1 ein geringfügig kleineres Volumen erreicht werden, für das PTV2 ergab sich jedoch aus diesem Konzept ein signifikant größeres Planungszielvolumen. Durch die Einbeziehung der postoperativer MR-Datensätzen in die Bestrahlungsplanung konnte der tumor- und ödembedingte midline-shift berücksichtigt werden. Ob die postoperative Konturierung nach den RTOG-Empfehlungen zur Verbesserung des Outcomes beiträgt, sollte durch weitere Verlaufsstudien untersucht werden. 
POS-24

\section{Erhebung der Fehler Sensibilität des online Dosis monitoring Systems IQM und des pretreatment QA Systems PTW Octavius bei Vmat Bestrahlungen}

\author{
M. Maffei ${ }^{1}$, S. Hofer ${ }^{2}$, P. Ferrari ${ }^{2}$, M. Haller ${ }^{2}$
}

${ }^{1}$ Dienst für onkologische Strahlentherapie des Südtiroler Sanitätsbetriebs, Bozen, Italien

${ }^{2}$ Dienst für med. Physik des Südtrioler Sanitätsbetriebs, Bozen, Italien

Einleitung: Es wurde ein MLC Fehler und ein Dosisfehler im Raystation Planungsystem anhand von drei Prostatabestrahlungsplänen simuliert. Dabei wurde bestimmt ab welcher Größenordnung der Fehler klinische Relevanz erreicht.

Anschließend wurde erhoben, ob die zwei QA Systeme in der Lage sind, diese Fehler mit den bei uns verwendeten Parametern zu detektieren

Methodik: Der MLC Fehler bestand darin, dass bei allen Control Points der Vmat Bestrahlungsbögen die Leafbank X2 nach aussen geschoben wurde.

Beim Dosisfehler wurden die Monitoreinheiten der einzelnen Segmente erhöht.

Sobald der Fehler drei Prozent Dosiserhöhung der Verschreibungsdosis ergab, wurde davon ausgegangen, dass er von seiner Größenordnung her klinisch relevant ist.

Nun wurde die Fehlerantwort der zwei QA Systeme bestimmt.

Beim IQM System wurde als Parameter für den Watch- und Alarmlevel 2 und $4 \%$ verwendet, bei der Gammaanalyse der PTW Octavius Messung $3 \%$ und $3 \mathrm{~mm}$.

Ergebnisse: Der IQM erreicht beim Verschub der Leafbank die Alarmschwelle von $4 \%$ Signalabweichung bereits bevor der Wert für die klinische Relevanz erreicht ist. Auch bei der PTW Octavius Messung wird die Alarmschwelle erreicht.

Beim Dosisfehler war die Sensibiltät der zwei Systeme etwas niedriger und sie waren beide knapp nicht in der Lage Alarm auszulösen bevor der Fehler klinisch relevant wurde. Dabei zeigte sich der PTW Octavius etwas sensibler als das IQM System.

Diskussion: Die beiden Systeme zeigten bei der Erhebung des MLC Leafbank Fehlers ausreichende Sensibiltät zur Fehlererkennung.

Bei der Erkennung des Dosisfehlers ergab sich eine etwas reduzierte Sensibilität.

\section{POS-25}

\section{Correlation between bone marrow dose volumes and acute hematological toxicity in rectal cancer patients treated with volumetric arc-therapy}

\author{
B. Aydin ${ }^{1}$, Z.L. Arican ${ }^{1}$, R. Kandemir ${ }^{2}$, B. Görken ${ }^{1}$
}

${ }^{1}$ Dokuz Eylul Universität Klinik für Strahlentherapie und

Radioonkologie, Izmir, Turkey

${ }^{2}$ Dokuz Eylul Universität Medizinische Strahlenphysik, Izmir, Turkey

Introduction: Acute hematologic toxicity is a common problem in patients receiving concurrent chemoradiotherapy (CRT) for treatment of rectal cancer. More than one-half of the body's bone marrow (BM) is located in the os coxae, sacrum, proximal femora, and lower lumbar spine. We compare different dosimetric parameters in rectal cancer patients receiving neoadjuvant radiochemotherapy and explore the incidence of hematological toxicity (HT) in these patients.

Methods: We analyzed 27 patients with rectal carcinoma undergoing treatment with oral capecitabine $(825 \mathrm{mg} / \mathrm{m} 2)$ and volumetric arc-therapy (VMAT) between March 2015 and December 2017. Pelvic bone marrow (BM) was contoured for each patient and divided into three subsites: lumbosacral spine, ilium, and lower pelvis. The volumes of bone marrow receiving $5,10,15,20,25,30,35,40,45$ and 50 Gy (V5, V10, V15, V20, V25, V30, V35, V40, V45 and V50, respectively) were calculated. The HT was graded according to the guidelines of the Radiation Therapy Oncology Group (RTOG) system. Multivariate regression models were used to test associations between dosimetric parameters and HT.

Results: RTOG grade $\geq 3$ lymphocyte toxicity was observed in 25 $(93 \%)$ patients. Increased lumbosacral V5-40 was associated with an increased Grade 3 or worse neutropenia $(p<0.01)$. Patients with increased BM-V10 and lower pelvis BM-V5-25 doses had higher rates of Grade 2 or worse lymphopenia ( $p=0.046$ and $p<0.04$ respectively). Bone marrow V50 and lumbosacral spine V5, V15 and V50 were associated with low hemoglobin levels $(p=0.04$ and $p<0.03)$. Dosimetric parameters involving the lumbosacral spine had stronger associations with HT.

Conclusion: The results of this study have shown an association between the volume of pelvic BM receiving low-dose radiation and acute HT in patients undergoing concurrent capecitabine $(825 \mathrm{mg} / \mathrm{m} 2)$ and VMAT. In radiotherapy treatment planning we recommend to keep the dose to the PBM as low as possible and BM receiving low dose radiation should be taken into account. 


\section{Autorenverzeichnis}

\section{A}

Altorjai G. POS-14, POS-21

Andreopoulos D. POS-01

Andrzejewski P. VAR-01

Arican Z. POS-25

Arnold C.R. VAR-02, VAR-06,

VAR-12, POS-05, POS-09

Artikan A. POS-01

Aschenbrenner B. MAM-04

Aydin B. POS-25

\section{B}

B. Hug E. VAR-11

Baier F. POS-14

Baltzer P. VAR-01

Beck F. POS-19

Belka C. MAM-01

Berger D. RTP-02, VAR-05, VAR-09

Böhler F. POS-13

Borneman J. POS-10

Braun M. MAM-01

\section{C}

Čemažar M. MAM-04

Chargari C. VAR-04

Clemens P. PRO-01, POS-13

Corradini S. MAM-01

\section{D}

Daniel M. VAR-01

de Vries A. VAR-07, POS-13

Deisenhammer T. RTP-01

Dejaco D. POS-05

Deutschmann J. POS-10

Dieckmann K. RTP-02

Ditz J. MAM-02

Dörr W. POS-04, POS-06,

POS-07, POS-08

Dragschitz F. VAR-01

Dunavülgy R. POS-11

Eiter H. VAR-07

Exeli L. VAR-11

\section{$\mathbf{F}$}

Fallast A. POS-20

Fastner G. POS-15, POS-16

Feichtinger J. VAR-08

Ferrari P. POS-24

Fetty L. PHY-01

Fokdal L. VAR-04

Freyschlag C. VAR-02

Fuchs H. PHY-01

Fuchs J. POS-19
Fussl C. VAR-10, VAR-11, POS-12

\section{G}

Gaasch A. MAM-01

Gamper E. POS-05

Ganswindt U. MAM-01, MAM-04, VAR-02, VAR-06, VAR-12, POS-09

Gastl R. POS-05

Geier M. PRO-01

Geinitz H. VAR-08

Georg D. VAR-01, PHY-01 POS-11

Georg P. VAR-10, VAR-11, POS-11, POS-12, POS-14

Goldner G. PRO-01, PRO-02, PRO-06, VAR-01

Gora J. POS-12

Gora J. PHY-02

Görken B. POS-25

Grimm C. VAR-09

Gruber M. PRO-01

Gruber N. VAR-10

\section{H}

H. Seewald D. H. PRO-01

Haller M. POS-24

Harasleben R. PRO-01, PRO-03

Harbeck N. MAM-01

Hawliczek R. PRO-03

Heilmann M. VAR-05

Helbich T. VAR-01

Helbich TH. POS-10

Herrmann H. VAR-05, POS-14

Hofer S. POS-24

Hofstätter S. RTP-02

Horninger W. VAR-06

Hug E. B. VAR-10, PHY-02, POS-11, POS-12, POS-14

\section{I}

Illedits S. POS-06 Ioannou L. POS-01

\section{J}

Jawadi N. POS-22, POS-23

\section{K}

Kadija D. POS-18

Kaiser J. POS-16

Kaltenegger C. POS-18

Kandemir R. POS-25

Kann T. PHY-02, POS-02
Kapp K. S. POS-03, POS-18, POS-22, POS-23

Kaufmann J. VAR-08

Kirisits C. VAR-05

Knäusl B. POS-12

Knoth J. VAR-09

Kocik L. VAR-08

Konrath L. VAR-10

Konstantinovic R. VAR-10, VAR-11, POS-12

Kopatz V. POS-06, POS-07

Kopp P. POS-16

Kovaiou O. VAR-06

Kowaliuk J. POS-04

Kowaliuk M. POS-07

Kowatsch M. PHY-03

Kragl G. POS-12, POS-14

Kragl G. VAR-11, PHY-02

Kranjc S. MAM-04

Kreuter C. POS-13

Kuess P. VAR-01, PHY-01, POS-04, POS-07

Künzler T. PHY-03

Kvitsaridze I. VAR-02, VAR-12, POS-09

\section{L}

Ladinig P. MAM-02

Langsenlehner T. PRO-01

Lindner A. VAR-06

Liu J. POS-10

Lövey K. MAM-02

Lukas P. VAR-02, VAR-06, VAR-12, POS-09

Lütgendorf-Caucig C. VAR-11, VAR-10, PHY-02, POS-11, POS-12

\section{M}

Maffei M. POS-24

Maier I. POS-10

Mangesius J. VAR-02, VAR-06, VAR-12, POS-09

Mazeron R. VAR-04

Meinschad M. PHY-03

Metz M. PRO-01, POS-19

Metz N. MAM-02

Mock U. VAR-10, VAR-11, POS-12

\section{$\mathbf{N}$}

Naschenweng C. MAM-02

Nechvile E. PRO-01

Negro G. MAM-04

Nesvacil N. VAR-05

Nesvacil N. VAR-04

Nevinny-Stickel M. VAR-02, VAR-12, POS-09
Nischelwitzer A. POS-20

Niyazi M. MAM-01

Notter M. MAM-03

Nout R. VAR-04

Nyholm T. VAR-01

0

Osztavics A. RTP-02

\section{P}

Padilla-Cabal F. PHY-01

Partl R. POS-03, POS-22, POS-23

Patsch JM. POS-10

Pazos M. MAM-01

Peratikou A. POS-01

Perpar A. VAR-10, VAR-11, POS-12, POS-14

Pichler R. VAR-06

Pirich C. POS-15

Polanec S. VAR-01

Poljanc K. PRO-03

Polterauer S. VAR-09

Pölzl A. POS-17

Porsch P. POS-15, POS-16

Pötter R. VAR-05, VAR-09

Poullos N. POS-01

Preihs M. POS-18

\section{$\mathbf{R}$}

Raditsch K. MAM-02

Regitnig P. POS-03

Reinthaller A. VAR-09

Reiterer J. PHY-02, POS-02

Reitz D. MAM-01

Resl C. PRO-01

Rettenbacher L. POS-15

Riedl D. POS-05

Ritschl H. POS-20

Ritt N. RTP-02

Ruegger PM. POS-10

Rumpold G. POS-05

\section{S}

Schallerbauer-Peter A. VAR-10

Schiebl M. MAM-02

Schiestl RH. POS-10

Schmid M. VAR-04, VAR-05, VAR-09, POS-14

Schmid R. VAR-05

Schmidt W. O. PRO-03

Schmidt-Erfurth U. POS-11

Schneider S. POS-21

Schönecker S. MAM-01

Schörghofer A. PRO-04, PRO-05 
Schratter-Sehn A.U. PHY-02, POS-02

Schreiner T. PHY-01

Schröder I. POS-07

Schuch S. PRO-03

Sedlmayer F. PRO-04, PRO-05, POS-15, POS-16

Seebacher V. VAR-09

Seel M. MAM-02

Seppi T. VAR-12

Skvortsov S. PRO-01, MAM-04, VAR-06

Skvortsova I. I. MAM-04

Soltner J. POS-20

Somay C. PRO-03

Sprenger M. POS-15

Stefanou S. POS-01

Steffal C. PRO-01, PHY-02, POS-02

Steffens P. POS-17, POS-22, POS-23

Stock M. VAR-11, PHY-02, POS-12, POS-14

Studnicka M. POS-16, POS-15

Sturdza A. VAR-09

Szeverinski P. PHY-03, POS-13

\section{$\mathbf{T}$}

Temmel U. POS-17, POS-18

Themos E. POS-01

Theodorou M. POS-01

Track C. VAR- 08

Trapp E. POS-03

Trifterer C. POS-18

Tubin S. VAR-03

\section{V}

Vasiljevic D. VAR-02, VAR-06, VAR-12, POS-09

Vaupel P. MAM-03

\section{W}

Wagner P. POS-19

Wass R. POS-15, POS-16

Weigel R. POS-09

Weiland B. VAR-12

Weingartner T. VAR-08

Westerveld H. VAR-04

Widder J. VAR-05, VAR-09, POS-14

Windischbauer S. POS-16

Wisgrill B. RTP-02

Wolf F. PRO-01, PRO-04, PRO-05, POS-16

\section{Z}

Zagler N. POS-19

Zehentmayr F. POS-16, POS-15

Zemora G. POS-08, POS-06

Zisser C. POS-17, POS-18

Zurl B. POS-17, POS-

18 , POS-20 